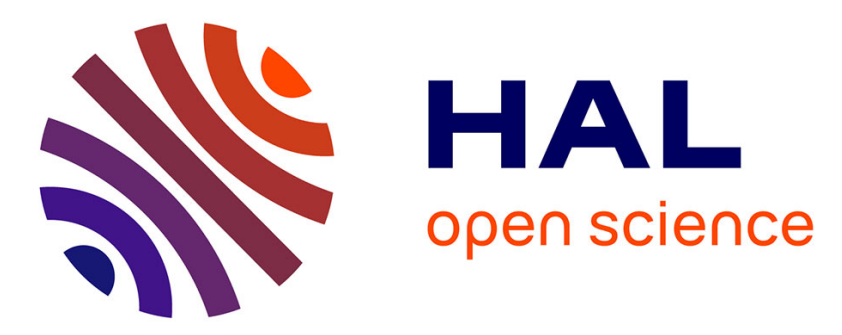

\title{
Photo-triggered polymer nanomedicines: From molecular mechanisms to therapeutic applications
}

Louis Beauté, Nathan D. Mcclenaghan, Sébastien Lecommandoux

\section{To cite this version:}

Louis Beauté, Nathan D. Mcclenaghan, Sébastien Lecommandoux. Photo-triggered polymer nanomedicines: From molecular mechanisms to therapeutic applications. Advanced Drug Delivery Reviews, 2019, 138, pp.148-166. 10.1016/j.addr.2018.12.010 . hal-02098559

\section{HAL Id: hal-02098559 \\ https://hal.science/hal-02098559}

Submitted on 8 Jul 2019

HAL is a multi-disciplinary open access archive for the deposit and dissemination of scientific research documents, whether they are published or not. The documents may come from teaching and research institutions in France or abroad, or from public or private research centers.
L'archive ouverte pluridisciplinaire HAL, est destinée au dépôt et à la diffusion de documents scientifiques de niveau recherche, publiés ou non, émanant des établissements d'enseignement et de recherche français ou étrangers, des laboratoires publics ou privés. 


\title{
Photo-triggered polymer nanomedicines: From molecular mechanisms to therapeutic applications
}

\author{
Louis Beautéa,b ${ }^{a}$ Nathan McClenaghan ${ }^{a^{*}}$, Sébastien Lecommandoux ${ }^{b^{*}}$ \\ a Institut des Sciences Moléculaires, Université de Bordeaux, UMR CNRS 5255, 351 Cours \\ de la Libération, 33405 Talence, France \\ b Laboratoire de Chimie des Polymères Organiques, Université de Bordeaux, Bordeaux \\ INP, UMR CNRS 5629, 16 Avenue Pey-Berland, 33607 Pessac, France
}

* Corresponding authors:

- Institut des Sciences Moléculaires, Université de Bordeaux / CNRS, UMR 5255, 351

Cours de la Libération, 33405, Talence, France

E-mail address:

- nathan.mcclenaghan@u-bordeaux.fr (N. McClenaghan)

- Laboratoire de Chimie des Polymères Organiques, 16, Avenue Pey-Berland, 33607

Pessac Cedex France,

E-mail address:

- lecommandoux@enscbp.fr (S. Lecommandoux) 


\section{Abstract}

The use of nanotechnology to improve treatment efficacy and reduce side effects is central to nanomedicine. In this context, stimuli-responsive drug delivery systems (DDS) such as chemical / physical gels or nanoparticles such as polymersomes, micelles or nanogels are particularly promising and are the focus of this review. Several stimuli have been considered but light as an exogenous trigger presents many advantages that are pertinent for clinical applications such as high spatial and temporal control and low cost. Underlying mechanisms required for the release of therapeutic agents in vitro and in vivo range from the molecular scale, namely photoisomerization, hydrophobicity photoswitching, photocleavage or heat generation via nanoheaters, through to the macromolecular scale. As well as these approaches, DDS destabilization, DDS permeation pore unblocking and formation are discussed. 


\section{Outline}

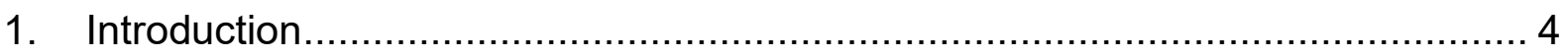

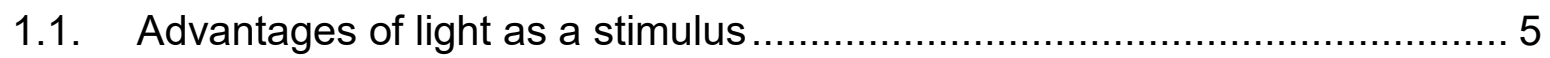

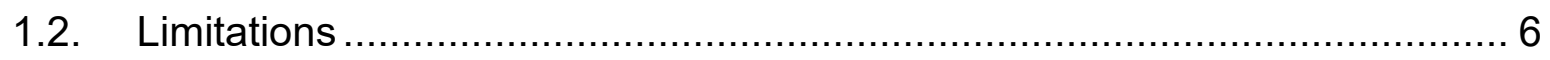

2. Molecular mechanisms of light-activated Drug Delivery System (DDS) ............. 7

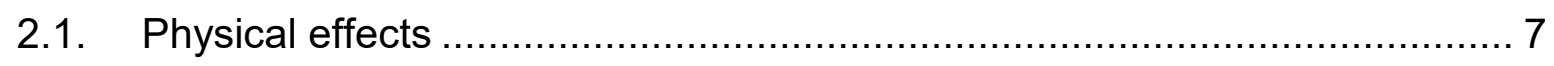

2.1.1. General mechanism for conversion of light energy to heat ................. 7

2.1.2. Design of nanoheaters for efficient light to heat energy transformation 8

2.1.3. Converting radiation: upconverting nanoparticles ............................ 10

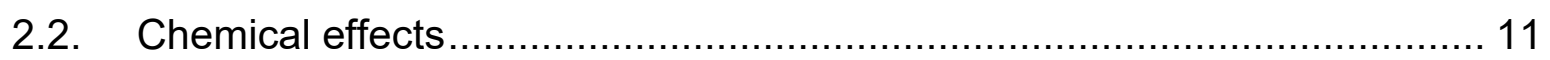

2.2.1. Photo-induced chemical cleavage ............................................... 12

2.2.2. Isomerization and rearrangements............................................... 15

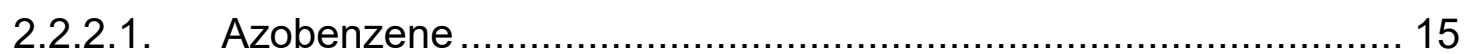

2.2.2.2. Hydrophobicity / Hydrophilicity switch molecules ........................ 17

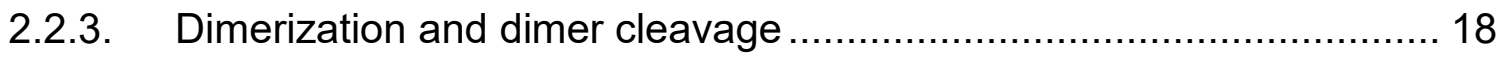

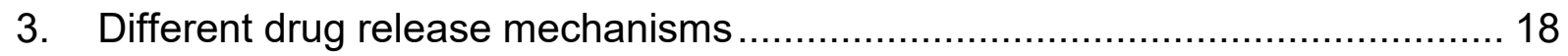

3.1. Light-induced structural changes inducing drug carrier destabilization ...... 18

3.1.1. Self-assembled nanoparticles .................................................. 19

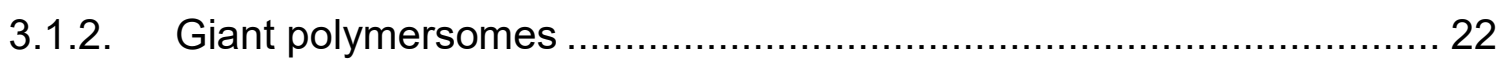

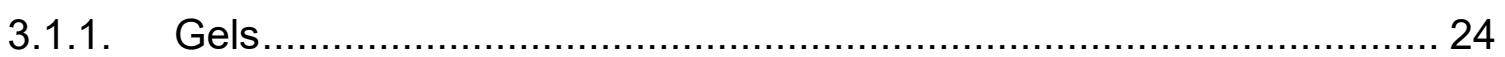

3.2. Light-induced increase of diffusion speed (Heat generation / permeability

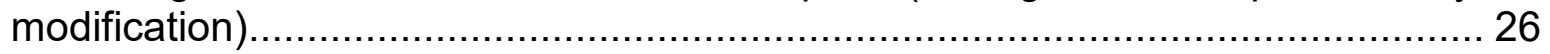

3.2.1. Polymer / Gel shrinkage (solubility change) ................................... 27

3.2.2. Glass transition and melting induced release .................................. 29

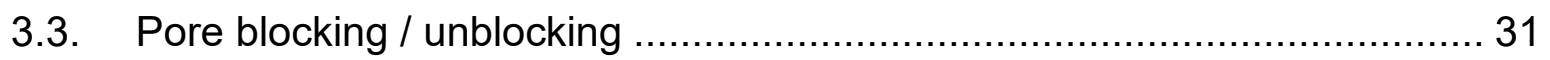

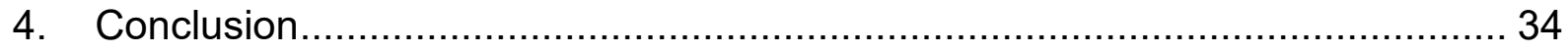

Keywords : Drug-delivery, light- responsive, polymers, nanomedicine, gels, nanoparticles 


\section{Introduction}

Nanomedicine is a relatively new field of biomedicine, which aims to use nanotechnology in order to find innovative systems for diagnosis and therapy. In recent years, nanomedicine has become a popular research topic, and rapid progress has been made in the quest for new systems that increase therapeutic molecule efficiency and reduce side effects. For this purpose, one major challenge is the development of efficient drug delivery systems (DDS). The efficacy of a treatment relies on the ability of the active substances to reach their site of action, at the right tissue or cellular level with the right concentration. When administrated, drugs are generally distributed throughout the body without any distinction between healthy and unhealthy organs. Such uncontrolled biodistribution is responsible for many side effects [1]. To reduce such undesired side effects, the dose is often lowered and the drug efficiency is not maximized. The development of strategies to overcome this problem by using DDS has been a challenging task for many years. Nanomedicine and nanoparticles for DDS represent a promising field to treat many diseases. Particles with nanoscale sizes present many advantages such as: they can be injected in the blood stream, a different biodistribution compared to therapeutic molecules, longer circulation in the blood stream and the possibility to reach organs without being invasive (compared to surgical methods). The ideal drug carrier should be stable, should not leak and increase the drug circulation time in the blood stream. Shear stress [2] [3] in the blood stream and a plethora of blood components may readily destabilize drug delivery systems. Also the drug delivery system should recognize the target, help active substances reach the target, accumulate next to or inside the target, and after endogenous or exogenous stimulus, release the drug to the right target [4]. Natural nanosystem structures and composition have served to inspire chemists in the design of efficient DDS, such as proteins or viruses. However, the possibility offered by natural materials is limited. Over the past decades huge advances in materials science, in particular in polymer science, have been made. Polymer properties and versatility were used in making a broad variety of robust, stimuli-responsive DDS. Moreover, the polymer can be tuned in order to be sensitive to different stimuli such as light. In this review only drug release mechanisms under light irradiation are considered, which we believe represents a promising and versatile class of exogenous trigger that can be remotely activated on demand. To meet all the requirements summarized above, active substances should be associated with a light sensitive carrier. In the first part of this review, advantages and limitations of light as a trigger will be presented then the effect of light at the molecular level will be discussed, together with the major mechanisms of action and their limitations. Finally, the effect of light activation when photoactive substances are incorporated into drug delivery systems will be considered. Scheme 1 summarized the light-driven processes considered herein, extending from the molecular level to the macroscopic level, resulting in drug release. 


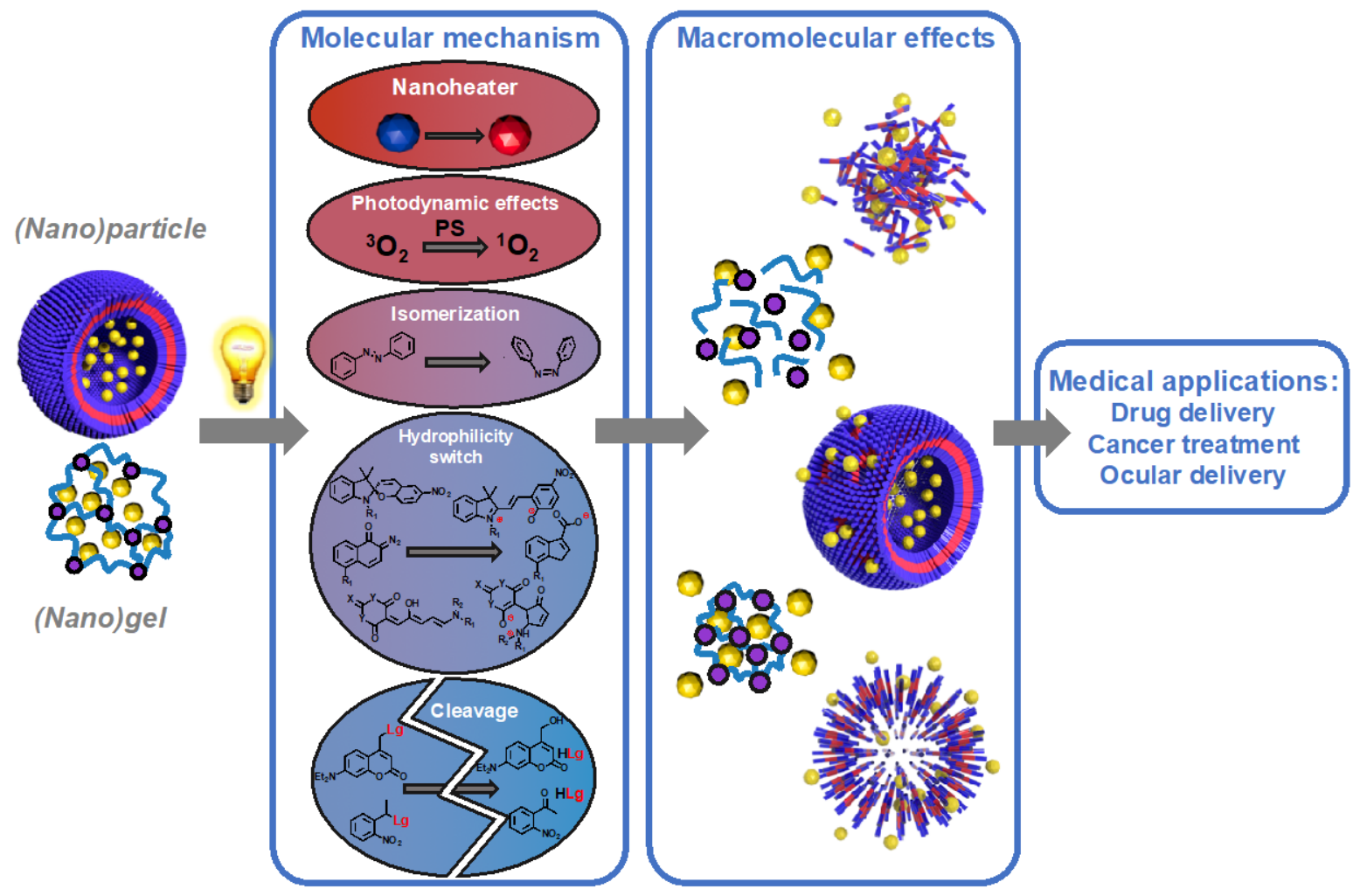

Scheme 1 Light-driven molecular processes responsible for drug release from nanocarriers.

\subsection{Advantages of light as a stimulus}

The use of light as a trigger for drug release presents many advantages compared to drug delivery systems based on a response to $\mathrm{pH}$, temperature, or enzymes [4]. Indeed, such drug delivery triggers are often poorly controlled as they depend on physiological parameters that can differ between individuals and are subject to local variations in the body. For example, temperature, $\mathrm{pH}$ or enzyme concentrations vary between persons, body parts, health and age. 
Additionally, light is an attractive trigger, which can be applied with a high degree of temporal and spatial accuracy, and whose intensity can be easily controlled. The light spectrum is wide and potentially allows triggering different systems at the same time.

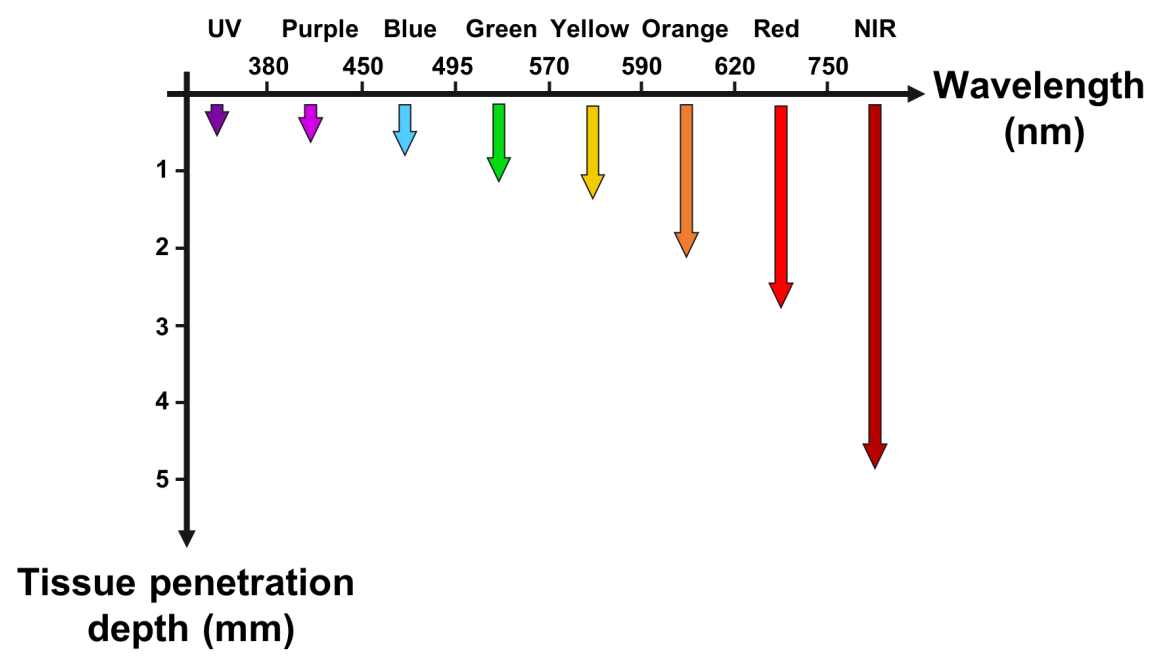

Fig. 1 General scheme of light depth penetration in tissue at several wavelengths for $1 \%$ transmittance (adapted from [5] [6])

The variety of light sensitive molecules is tremendous and light excitation can target a precise location in the body during a specific time. For drug delivery applications, light is non-invasive, easy to produce and not expensive. More specifically, near infrared light is particularly interesting, as it offers the advantage of being less harmful for cells than UV [7] [8], and can penetrate deeper into the tissue [9] (Fig. 1). Globally, it is clear that light sensitive systems for drug delivery can be presented as promising tools with important future development.

\subsection{Limitations}

While the light spectrum is broad, only a narrow range of wavelengths can interact at the molecular level. UV light, typically in the range $254-400 \mathrm{~nm}$, is highly energetic that can provoke a variety of photoreactions with high efficiency. Therefore, most light-sensitive molecules are UV-sensitive and light-triggered systems that have been developed for drug delivery applications are also mostly UV-sensitive. The primary disadvantage with UV light is that it cannot be applied to human subjects because of adverse physical effects (more specifically with UVC and UVB from $254 \mathrm{~nm}$ to 360 $\mathrm{nm}$ ). Indeed, it is well established that UV damages the skin: it induces molecular damage on lipids, nucleic acid and proteins leading to cellular injury and skin cancer [10]. Also, the UV penetration depth is small, limiting its effect to the superficial skin layers [11]. As such, for drug delivery applications, it would be difficult to reach and deliver payloads to internal organs. On the other hand, light in the infrared and nearinfrared (IR and NIR) is a very interesting alternative to UV, since it can go much 
deeper into the tissues and is less dangerous for cells. Nevertheless, IR is not as energetic as UV and as such cannot drive as many reactions. These limitations can be overcome in two different ways that will be described in details in the following sections: (i) combining IR-sensitive systems that generate heat with temperaturesensitive systems [12] [13] [14] [15] [16], and (ii) by using upconverting nanoparticles, which can convert IR to UV [17] [18] [19] [20].

\section{Molecular mechanisms of light-activated Drug Delivery System (DDS)}

\subsection{Physical effects}

Light can induce two major physical effects: (1) increasing the system temperature and (2) converting radiation to other wavelengths. In both cases light energy can be used to promote drug release.

\subsubsection{General mechanism for conversion of light energy to heat}

It is well known that light can produce heat, especially when interacting with some nanomaterials, which can be used to directly kill cells. This principle is referred to as photothermal therapy. In this review, this mechanism for therapy will not be discussed as our main subject of interest concerns systems that deliver payloads thanks to the heat generated upon irradiation. In this part, materials able to convert light energy to heat energy, deemed "nanoheaters", will be described.

In order to convert light energy into heat energy, several photothermal agents or nanoheaters can be used such as noble metals, transition metal dichalcogenides, carbon nanotubes (CNT), graphene oxide (GO) dye molecules and semiconductors (organic or inorganic). These photothermal agents absorb light and consequently their electrons become excited. The energy can be dissipated via non-radiative decay channels and heat the immediate environment. Dye molecules such as indocyanine green or naphtalocyanine have strong absorption nevertheless they undergo photobleaching, thereby reducing their efficacy. Noble metals do not undergo photobleaching and can have absorption cross sections four to five orders of magnitude larger than dye molecules [21]. Electromagnetic waves interact with electrons on noble metal surface [22]. Indeed, these materials have free electrons on their surface that can oscillate with electromagnetic field. This phenomenon is called Surface Plasmon Resonance (SPR) [23]. Materials with SPR properties are, for instance, noble metals, transition metal dichalcogenides, carbon nanotubes (CNT), graphene oxide (GO) and semiconductors (organic or inorganic). At the appropriate electromagnetic resonance, the energy transfer is maximum [24]. The photon excites the electron and the energy is absorbed. The electron is then thermalized, the energy is transferred to the particles, creating heat that is then transferred to the surrounding medium. For metallic particles, their size and shape can be easily tuned to modulate 
and control the resonant wavelengths [25].

\subsubsection{Design of nanoheaters for efficient light to heat energy transformation}

There are a wide variety of materials that can transform light energy to heat energy and that have been developed over the past years. We will focus here on systems that are sensitive to NIR because they are the most applicable for clinical development.

The most popular nanoheaters are probably the noble metals like gold, silver, platinum or palladium. They have varying optical properties, they are easy to tune and to functionalize [26]. These properties make them very interesting for drug delivery systems. In addition, some organic molecules can also convert light into heat, such as porphyrin [27] or trisodium salt copper chlorophyllin (488 nm) [28]. Moreover, 2D materials have attracted a great interest recently for drug delivery applications because: they have a very large specific surface area so they absorb efficiently NIR and can adsorb therapeutic molecules with aromatic character, such as doxorubicin [29] or chlorin e6 [30] due to $\pi-\pi$ stacking. As an example graphene oxide and transition metal dichalcogenide are 2D materials which have been used as drug delivery systems [29] [30]. A recent review on stimuli responsive graphene oxide emphasized the interest of this kind of drug delivery system [31]. 2D transition metal dichalcogenides have also been recently reviewed [32]. One of them, $\mathrm{MoS}_{2}$ possesses lower cytotoxicity than graphene oxide, which is better suited for biomedical applications.

Carbon nanotubes (CNT) have other advantages compared to 2D materials. Unlike nanosheets, CNT possess an intra-tube empty space where they can encapsulate a wider variety of drugs (Fig. 2) [33]. Their absorption wavelengths are constant and do not depend on their lengths or size, contrary to gold nanoclusters [25]. Nevertheless, several problems should be overcome for biomedical applications, such as residual impurities: metal traces, small fullerenes amorphous carbon or their low solubility in aqueous solvent [34]. Also even if several drugs have been encapsulated inside CNTs [34], the only example, from our knowledge of releasing drug from their intratube empty space thanks to light is described in this review.

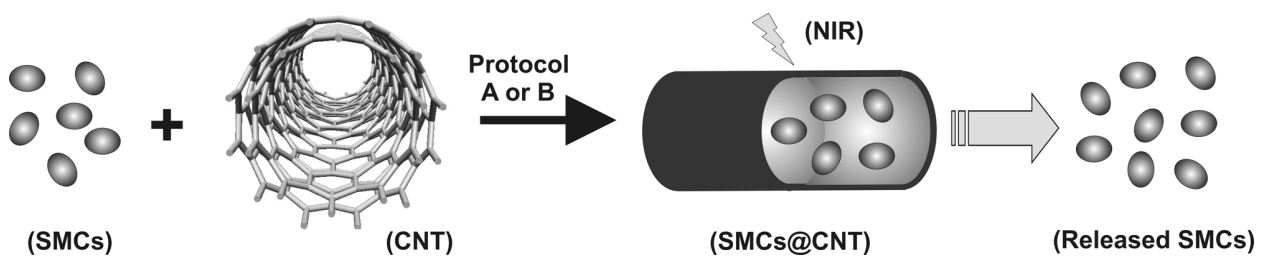

Fig. 2 Indole release from carbon nanotube after IR irradiation [33] [Reprinted with permission from Z. Su, S. Zhu, A.D. Donkor, C. Tzoganakis, J.F. Honek, Controllable Delivery of Small-Molecule Compounds to Targeted Cells Utilizing Carbon Nanotubes, J. Am. Chem. Soc. 133 (2011) 6874-6877. Copyright (2011) American Chemical Society.]

Another type of nanoheater is based on inorganic and organic semiconductors, which 
are attracting attention for cancer treatment [35]. Indeed, they show a broad absorption in the NIR region and good photothermal conversion. They also are stable in vivo, easy to synthesize, low cost and insensitive to photobleaching. Huang et al. reported a system with $\mathrm{Cu}_{1.75} \mathrm{~S}$ coated with a thermosensitive polymer, which was able to efficiently release doxorubicin under NIR light (808 nm) [13].

Organic semiconductor polymers were also proposed as a recent class of nanoheater. These polymers are better known for their application in organic electronics but some of them can also absorb in the NIR region. They are easy to synthesize, flexible, tunable, have a better biocompatibility [36]. A polymeric semiconductor poly(diketopyrrolopyrrole-alt-3,4-ethylenedioxythiophene) and pluronic 127 formed nanoparticles with a hydrodynamic diameter of $10 \mathrm{~nm}$, that were successfully integrated in a poly(N-isopropylacrylamide) (pNIPAAM) gel. This nanoparticle / hydrogel system was demonstrated to release doxorubicin on demand under NIR light (808 $\mathrm{nm}$ ) to cells in vitro [37]. Another study uses the second NIR window to penetrate the skin [38]. A polymeric semiconductor poly(bis(5oxothieno[3,2-b]pyrrole-6-ylidene)benzodifurandione)-co-poly(bithiophene) which absorbs NIR light between 1000 and $1350 \mathrm{~nm}$ and converts it to heat was synthesized [39]. This organic semiconductor was self-assembled with an amphiphilic copolymer into micelles (diameter of $45 \mathrm{~nm}$ ). These NIR sensitive micelles were then used to kill cancer cells due to the heat generated by the $1064 \mathrm{~nm}$ irradiation.

All the aforementioned particles are potentially promising for drug delivery, but alone they are often insoluble or not biocompatible. The association of nanoheater with functional and biocompatible polymers is symbiotic as it allows combination of advantages of both systems: light sensitivity and thermosensitivity (Fig. 3).

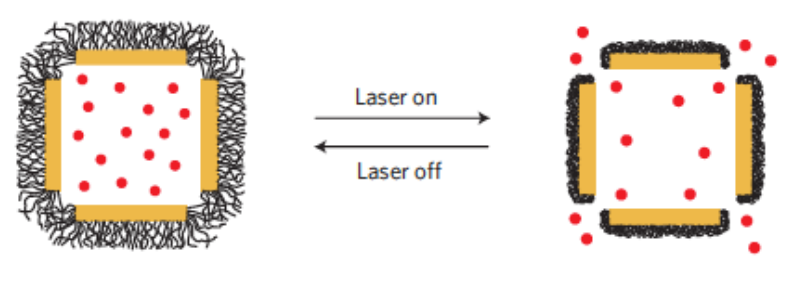

Fig. 3 Representation of gold nanocages coated with thermosensitive polymer chains (pNIPAAM-co-pAAM) which release doxorubicin under NIR radiation [14] [Reprinted by permission from Springer nature, nature materials M.S. Yavuz, Y. Cheng, J. Chen, C.M. Cobley, Q. Zhang, M. Rycenga, J. Xie, C. Kim, A.G. Schwartz, L.V. Wang, Y. Xia, Gold nanocages covered by smart polymers for controlled release with near-infrared light, Nat. Mater. 8 (2009) 935-939. Copyright (2009)]

A wealth of polymers can be tuned to be thermoresponsive, biocompatible and biodegradable [40] making them suitable as thermosensitive systems for drug delivery [41]. Grafting polymers can confer thermosensitivity, biocompatibility, and water solubility to the nanoheater. Polymers combined with nanoheaters can be used as a medium to encapsulate drugs. The wide variety of polymer and nanoheater offer tremendous possibilities to develop innovative new drug carriers. 
As an elegant example of combining IR-sensitive systems that generate heat with temperature-sensitive systems, $\mathrm{N}$-isopropylacrylamide (NIPAAm) and gold nanoparticles were combined [12] In this article nanoshell gold nanoparticles of around $20 \mathrm{~nm}$ were incorporated in temperature sensitives co-polymers hydrogel nanoparticles : NIPAAm and acryl-amide (AAm). Irradiation by NIR light (1064 nm) of the particles could enhance the release of methylene blue or model protein (bovine serum albumin).

\subsubsection{Converting radiation: upconverting nanoparticles}

Upconversion is a phenomenon that converts low energy radiation into high energy radiation (e.g. NIR photons into visible and UV photons). In drug delivery applications these particles offer access to use of a wide variety of UV-activated molecules [42]. The upconversion mechanism involve two ions: one that absorbs the incident light called sensitizer ion, and another that emits the energy absorbed called activator ion. Several mechanisms with one or several sensitizers ions and one activator ion can result in the conversion of NIR into visible and UV light [17]. Here we describe the case where the sensitizer ion and the activator ion are the same. In this case ions that can upconvert radiation can absorb two or more low energy photons (Fig. 4, h $v_{1}$ and $h v_{2}$ ) and subsequently emit one high energy photon (or transfer the culminated excitation energy $\mathrm{h} v_{3}$ ). To do so, these ions need to have excited states, which are optically active and have a long-excited state lifetime $\left(E_{1}\right)$. The energy is accumulated and released in the form of one high energy photon. Upconverting nanoparticles (UCNPs) are composed of a crystalline host and a dopant, usually trivalent lanthanide ions, such as $\mathrm{Lu}$, La, $\mathrm{Pr}, \mathrm{Nd}, \mathrm{Sm}, \mathrm{Eu}, \mathrm{Gd}, \mathrm{Tb}, \mathrm{Dy}, \mathrm{Ho}, \mathrm{Er}, \mathrm{Tm}$. The most common lanthanides used are $\mathrm{Er}^{3+}, \mathrm{Tm}^{3+}$, and $\mathrm{Ho}^{3+}$. These inorganic ions have a lot of advantages such as very low background fluorescence, narrow emission band width, high resistance to photobleaching, and their absorbance wavelengths allow deep penetration into the tissues [43].

To illustrate the use of upconverting nanoparticles, a study used poly(ethylene oxide)-block-poly(4,5-dimethoxy-2-nitrobenzyl methacrylate) as a UV sensitive copolymer [18]. The copolymer formed $100 \mathrm{~nm}$ micelles and UCNPs were incorporated inside the self-assemblies during their formation. Under NIR irradiation $(980 \mathrm{~nm})$ the upconverting nanoparticles converted the wavelengths into visible and UV light directly inside the micelles. As a consequence o-nitrobenzyl groups were cleaved and the particles were destabilized. The disruption of the micelles induced Nile red release.

As another example, UCNPs composed of $\mathrm{NaYF}_{4}, \mathrm{Y}: 25 \%$ and $\mathrm{Tm}: 0.5 \%$ were synthesized with diameter of 20-30 nm [19]. A copolymer of hydrophilic pNIPAAM and hydrophobic poly(acrylate) containing spiropyran, was self-assembled on the surface of UCNPs (115 nm in diameter). Spiropyran becomes hydrophilic under UV light. These hybrid nanoparticles were irradiated with NIR $(980 \mathrm{~nm})$, UCNPs 
converted this radiation into UV and visible light (360 $\mathrm{nm}$ and $470 \mathrm{~nm}$ ), spiropyran absorbed UV radiation and became hydrophilic, resulting in particle destabilization and coumarin 102 release.

Another elegant study used UCNPs and UV sensitive ortho-nitrobenzene (ONB) as the photocleavable moiety. Jalani et al. used lanthanide-doped UCNPs that were coated with hydrogel [20]. The inorganic nanoparticles used have a rhombus plate shape, an average length, width and thickness of $78 \mathrm{~nm}, 52 \mathrm{~nm}$ and $7 \mathrm{~nm}$, respectively. These UCNPs can convert NIR into UV radiations (347 and 362nm). To form the hydrogel layer, chitosan was grafted on the UCNPs surface and then crosslinked thanks to a photocleavable ONB-PEG-ONB functional oligomers. A fluorescent protein FITC-BSA was loaded in the hydrogel layer. Then the nanoparticles were irradiated with NIR, that was converted into UV radiation. Consequently, the UV radiation cleaved the ONB moiety, the nanogel disintegrated and released FITC-BSA protein.

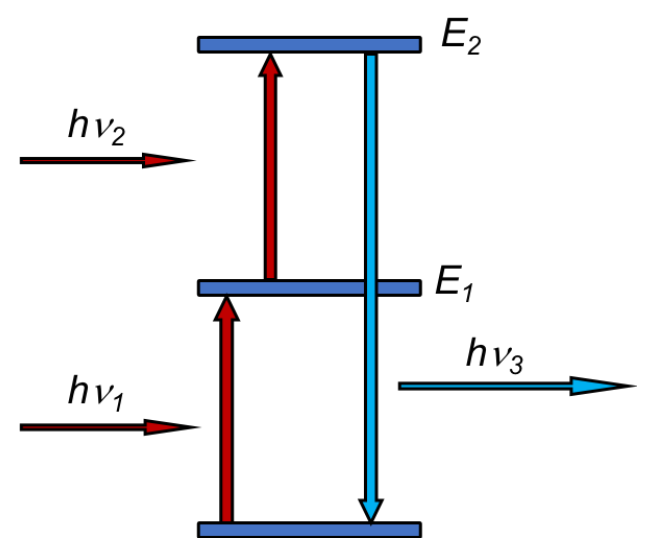

Fig. 4 Schematic representation of an upconverting energy diagram. Energy state $E_{1}$ is optically active and has a long-excited state lifetime. $E_{2}$ is the energy state from which the energy transfer can occur.

\subsection{Chemical effects}

As previously described, considering drug delivery applications, photons have to penetrate deeply through the skin, and NIR light is therefore preferable. The problem is that most light-sensitive molecules that undergo chemical modification after irradiation are sensitive to UV. It is thus critical to develop molecules, which absorb visible or NIR light. An interesting review on molecules sensitive to UV or NIR highlights their diversity [44]. The conundrum of low energy photons versus higher energy processes can be also overcome, thanks to upconverting nanoparticles. As these particles convert IR into UV, the use of a wide variety of UV sensitive molecules for drug delivery applications can be enabled [20]. Therefore, both NIR and UV sensitive molecules are presented in the current review. In this part the 
diversity of available molecules for drug delivery application will be presented. These molecules react in three different ways as a response to incident light: cleavage, isomerization or rearrangement and cross-linking.

\subsubsection{Photo-induced chemical cleavage}

Two main families of cleavable molecules based on coumarin (Fig. $5 \mathrm{~A}$ ) and ONB (Fig. 5 B) have been developed.
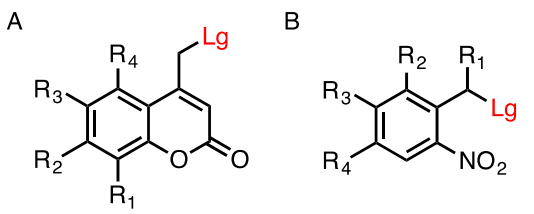

Fig. 5. The two main cleavable molecules used for drug delivery, Lg is the leaving group A) Coumarin and possible substitutions, $B$ ) ortho-nitrobenzene and possible substitutions.

The design of efficient cleavable molecules requires the presence of good leaving groups in their structure. In addition, the product formed after irradiation should be stable in order to avoid any recombination and increase the cleavage efficiency. Therefore leaving groups often used are carboxylic acid, carbamate or carbonate [45].

The ortho-nitrobenzene family is probably the most popular cleavable group used in the past years. In particular o-nitrobenzyl (ONB) derivatives have been widely developed for drug delivery applications despite some major drawbacks. Indeed, their release rates are slower than those of coumarin derivatives [46]. In addition, after irradiation, nitrosoaldehyde is formed and can react with amines to form an imine bound, which can especially be a problem for the surrounding proteins. Also nitrosoaldehyde can degrade to form brown compounds which act as an internal filter, thus decreasing the ONB cleavage efficiency [47] and can be potentially toxic [48].

A lot of ONB analogues have been synthesized in order to improve the cleavage efficiency, side product formation, solubility, or to tune irradiation wavelength [45]. Nevertheless, the substitution nature and position cannot predict the trend on photoreaction efficiency. Surprisingly, these analogues have been rarely used for drug delivery applications and most of these systems use the classic ONB without substitutions [49] [50] [51] [52] [53] [54]. To improve the ONB properties two main chemical substitions were developed on the benzylic carbon and the aromatic ring. The benzylic position substitution corrects one drawback: the formation of the aldehyde, which leads to imine formation with an amine. Several moieties have been tested, especially electron withdrawing moieties. Trifluoromethyl on the benzyl position increases the quantum yield from 0.13 to 0.7 [55]. The methyl group also avoids aldehyde formation and increases the quantum yield to 0.64 (in MeCN) [56] and this derivative has been used in drug delivery applications [20] [57] [58]. 
Substitution on the aromatic ring has also been studied. It has been evidenced that the presence of one nitro group on the second ortho position increases the quantum yield significantly. One interesting feature of this kind of substitution is to change the absorption wavelengths. As such, the substitution with O-nitro-dibenzofuran increases the absorption wavelength maxima [58] and the introduction of two methoxy groups allows release of leaving groups at $405 \mathrm{~nm}$ (Fig. 6 A) [59]. Another class of o-nitrobenzene is o-nitrophenethyl group (Fig. 6 B). These compounds with a methyl on the benzylic position present a relatively high quantum yield: 0.35. A new class of o-nitrophenethyl group has been synthesized which increases the absorptivity and the release rate (Fig. $6 \mathrm{C}$ and D) [60].

Coumarins constitute another important class of photochemically-active molecules, which can be used both as a crosslinker and as a cleavable group. It has numerous advantages and it is a good substitute to ONB, especially because of its faster release rate compared to ONB [46], its efficient release of a wide variety of leaving groups, and because it has important molar absorption coefficient with a larger absorption than ONB ( $\lambda_{\max }$ from $310 \mathrm{~nm}$ to $490 \mathrm{~nm}$ ). Depending on their substituent, coumarin derivatives that are released as a byproduct, can be toxic [61] or can have beneficial effects [62]. Coumarin derivatives can be easily tuned to adapt their toxicity. A lot of analogues have been synthesized to improve solubility or increase its absorption wavelengths and easy structure modifications shift the absorption to the red [45]. As an example, 7 -aminocoumarin has a $\lambda_{\max }$ between $350-$ $400 \mathrm{~nm}$ with a relatively good quantum yield: $0.21-0.28$. Its relative efficient release made this coumarin derivative an interesting candidate for drug delivery application and it has been used many times for this purpose [63] [64] [65] [66]. Feringa and his group covalently attached an antibiotic to the coumarin derivatives and after UV irradiation $(380 \mathrm{~nm})$ the antibiotic was released in order to control the bacterial population.

Another example used coumarin as the light sensitive moiety in conjunction with well-defined hollow mesoporous silica particles (HMS) [65]. The particles (250 nm in diameter) were loaded with doxorubicin. In order to obstruct pores and avoid undesired doxorubicin release, a copolymer containing coumarin groups was self-assembled and loaded with HMS to form a layer that enveloped the particles. This copolymer was synthesized from 2-hydroxyethylacrylate (HEA), methacrylamide hydrochloride (APMA) and 7-(didodecylamino) coumarin-4-yl]methyl methacrylate (DDACMM). A folate group was grafted on APMA, for selective cancer targeting and poly(DDACMM) is a poly(methacrylate) bearing hydrophobic coumarin derivatives. This polymer is both hydrophobic and light sensitive. The coumarin cleavage with UV or NIR light (365 nm or $800 \mathrm{~nm}$ with two photon absorption) made the copolymer layer hydrophilic, resulting in its solubilization in the medium. The pores were then open and as a consequence doxorubicin trapped in the hollow sphere was released. In order to red shift the absorption wavelength and improve the compatibility of radiation with cells, new coumarin molecules have been described. Three molecules with interesting photochemical properties have been identified: red shifted absorption with maximum absorption $\left(\lambda_{\max }\right)$ up to $487 \mathrm{~nm}$ and significant release rate with blue- 
cyan light [67]. Electron-withdrawing groups were introduced at the coumarin 2- and 3- positions such as sulfur or cyano groups. In Fig. 6, coumarin derivative $E$ has $\lambda_{\max }=472 \mathrm{~nm}, \mathrm{~F}$ has $\lambda_{\max }=443 \mathrm{~nm}$ and $\mathrm{G}$ has $\lambda_{\max }=487 \mathrm{~nm}$.

One side-reaction was reported as a drawback for the release of a thiol where the molecule underwent an isomerization instead of a cleavage [58]. The goal of the study was to protect cysteine on a peptide with a coumarin photo-removable group. Instead of thiol cleavage after irradiation, the coumarin isomerized and the thiol group was grafted on position 2 . The final product is not photocleavable and consequently, the protecting group cannot be removed.

$A$<smiles>COc1cc(C(C)C)c([N+](=O)[O-])cc1OC</smiles>

C<smiles>CCC(C)c1cc(C(=O)c2ccccc2)ccc1[N+](=O)[O-]</smiles>

E<smiles></smiles>

B<smiles>[R3]c1cc([N+](=O)[O-])c(C([R8])C[Hg])c([R6])c1[2H]</smiles><smiles>CCc1cc([N+](=O)[O-])c(C(C)CCl)cc1Sc1ccccc1</smiles>

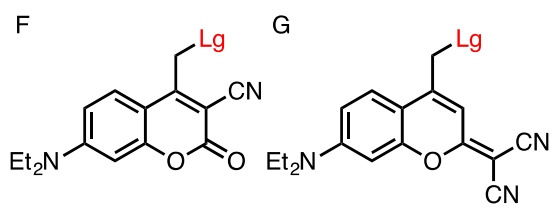

Fig. 6 Light-sensitive compounds that undergo cleavage under irradiation. These molecules have been designed in order to red shift their absorption. A) ONB with two methoxy groups B) o-nitrophenethyl and possible substitutions $($ ) and D) o-nitrophenethyl derivatives with better release rate and absorptivity E) F) and G)

Coumarin derivatives with significant release with blue-cyan light

In this part, we mainly reported two main families of photocleavable molecules based on O-nitrobenzyl and coumarin. They have been widely studied and their properties were greatly improved to optimize their development for biological applications, especially in the drug delivery field. In addition to these important derivatives, other cleavable molecules have been reported. A summary of these different classes of photocleavable molecular building blocks is presented in table 1.

Table 1. List of the main important classes of photocleavable molecules that have been developed for biomedical applications in recent years.

\begin{tabular}{llll}
\hline Molecules & $\lambda_{\text {irra }}(\mathbf{n m})$ & Quantum yield & Examples \\
\hline O-nitrobenzyl & & & [20] [49] [50] [51] [52] [53] [54] \\
{$[57][58]$} & & \\
$254-365[45]$ & $0.0013-0.7[45]$ & \\
[63] [64] [65] [66] [67] [68]
\end{tabular}




\section{Ruthenium polypyridine}

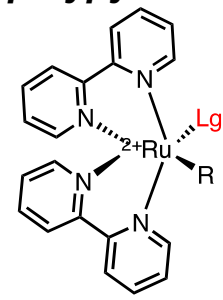

\section{BODIPY}<smiles>Cc1c(C)c(C)n(C(C)C)c1C</smiles>

450 to $575[72]$

0.15 in methanol

[72][73] not been used for drug delivery systems.<smiles></smiles>

$300-375[74]$

0.22 for phosphate release in $\mathrm{MeOH}$ [75]

[74]

$0.072-0.093$ in

$300-450[76]$

$\mathrm{H}_{2} \mathrm{O} / \mathrm{MeCN}$ for alcohol

[53] and carboxylic acid [77]

[78]<smiles>[R5]NC(=O)C(C)(C)NC(C)(C)NC(=O)C(C)(C)NC</smiles>

$365[78]$

amino-1,4benzoquinone<smiles>CCC1=CC(=O)C(N2CCCC2)=CC1=O</smiles>

$590[79]$

$0.003-0.007$ in $30 \%$ aq. $\mathrm{CH} 3 \mathrm{CN}$ [79]

[79]

\subsubsection{Isomerization and rearrangements}

\subsubsection{Azobenzene}

Azobenzene is a popular molecular switch that has been used in drug delivery systems because it can be efficiently and reversibly isomerized between transand cis- forms. Indeed, upon UV irradiation (365 nm) the thermodynamically stable trans- form isomerizes into the cis- form. This isomer is metastable and has a substituent-dependent half-life of up to two days [80]. If irradiated with visible light $(450 \mathrm{~nm})$, azobenzene quickly returns to the trans- isomer [81] (Fig. $7 \mathrm{~A}$ ). 

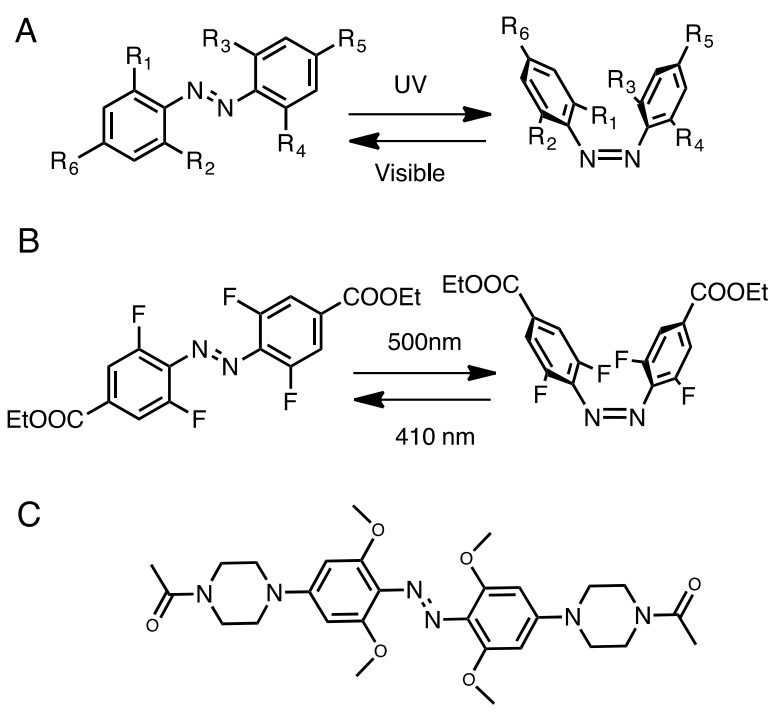

Fig. 7 A) Reversible azobenzene trans-cis isomerization under UV and visible light B) o-Fluoro-azobenzene showing a red shift in absorption and a stable cis isomer ( $\tau_{1 / 2}=2$ years) [82] C) Azobenzene derivative showing particularly large red shifting [83].

This molecular switch is reversible over multiple cycles without deterioration. In the drug delivery field this transformation is useful in inducing polymer or peptide property changes [84]. To improve the release, a range of derivatives has been developed to tune two major photochemical properties of azobenzene: the absorption wavelengths required to switch between the cis- and trans- forms and the isomer's thermal stability. For in vivo applications, light absorption should be in the near infrared in order to go deeply through the skin. In addition, thermal stability, characterized by the half-life of the less thermodynamically stable form, can be tuned depending on the system needs. A short half-life provides a fast back isomerization in the dark and an efficient on/off switch. A long half-life assures longterm system modifications.

Substitutions on the para- and ortho- positions have also been widely explored [85]. The para- position is often used to attach molecules of interest such as peptides or polymers [86]. The ortho- position red-shifts the absorption and tunes the thermal stability of the two isomers. A wide variety of groups have been tested including the amino [87] and chloro group [88]. Substitutions with four fluorines at the ortho- positions showed a significant red-shifting and an improvement in the thermal stability [82]. The irradiation of the trans- form with green light $(>500 \mathrm{~nm})$ showed $90 \% \mathrm{Z}$ isomerization and a half-life of circa 2 years (Fig. $7 \mathrm{~B}$ ). Substitution with 4 o-methyl groups showed significant red-shifting, with absorption up to $680 \mathrm{~nm}$ [83] (Fig. 7 C). Unfortunately these molecules are sensitive to reduction by glutathione which can be a problem for drug delivery applications [84]. The tetra-ortho-methoxy-substituted azobenzene has been used to release doxorubicin with red light [89]. These azobenzene derivatives show a great potential for drug delivery due to their long absorption wavelengths and excellent fatigue resistance. 


\subsubsection{Hydrophobicity / Hydrophilicity switch molecules}

Certain molecules undergo hydrophobicity / hydrophilicity switching upon irradiation, which can be either reversible or irreversible. Three molecular derivatives were identified for drug delivery applications: spiropyran [90], 2-diazo1,2-naphthoquinone (DNQ) [91] [92] and more recently donor-acceptor Stenhouse adducts (DASAs) [93]. These molecules can be incorporated into polymers, for example on the polymer backbone. After switching they can induce a significant change in the polymer property such as polymer hydrophobicity. This property is a key factor for some nanoparticle or gel stability. Changing the property at the molecular level can change the property at the macromolecular level and thus destabilize gels or nanoparticles [94] [95] [96] [97].

Under UV light, the closed ring form of spiropyran undergoes a $\mathrm{C}-\mathrm{O}$ bond cleavage, resulting in the zwitterionic merocyanine form. The merocyanine can reversibly lead to the hydrophobic ring-closed form under blue light (420 nm) (Fig. 8 A). Spiropyran is biocompatible and can be easily grafted onto any matrix. Even if the response is fast, this molecular switch presents one major drawback, as after several switch cycles the molecule undergoes photodegradation [98].

A

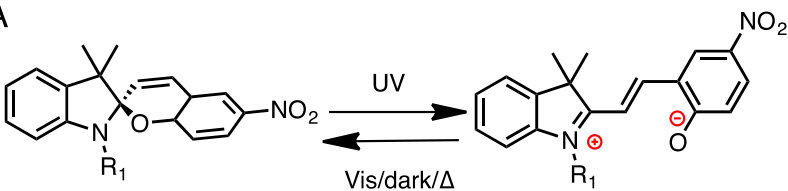

B

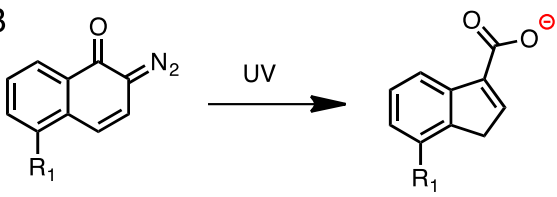

C

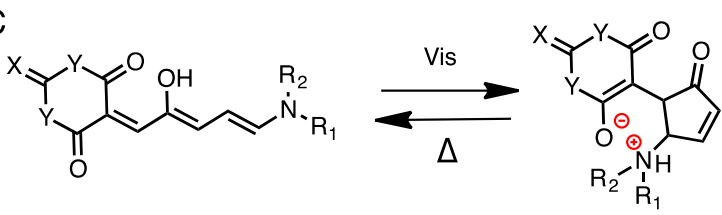

Fig. 8. Switch between hydrophobic isomer and hydrophilic isomer A) Spiropyran and merocyanine forms, B) Irreversible DNQ rearrangement induces hydrophilicity C) DASA molecular switch inducing hydrophilicity, $\mathrm{Y}=\mathrm{O}, \mathrm{NCH}_{3} ; \mathrm{X}=\mathrm{O},\left(\mathrm{CH}_{3}\right)_{2}$

DNQ has attracted a lot of attention because of its ability to act as a hydrophilicity switch that can be activated by UV or by NIR light in a two photon process [91] [92] [95] [97] [99]. Under these radiations DNQ undergoes an irreversible Wolff rearrangement [100] leading to a carboxylic acid with a pKa of 4.5 [101] (Fig. 8 B) that is charged and thus hydrophilic in physiological conditions $(\mathrm{pH}=7.4)$. DNQ has been tested on cells and was interestingly shown to be cytocompatible [95] [97]. DASA is a relatively new class of molecules that have been developed for hydrophilicity switch that present interesting features for drug delivery applications. DASA photoswitches under visible light (Fig. $8 \mathrm{C}$ ), the reaction being reversible and 
displaying excellent fatigue resistance [93]. The two forms have important property differences. Without illumination, the molecule is hydrophobic and compact, and becomes zwitterionic and extended after irradiation. These differences lead to efficient particle destabilization for cargo release [94]. This new class of molecules has not yet been widely exploited for drug delivery. One example reported the incorporation of DASA in the backbone of an amphiphilic copolymer [102]. To this end PEG was used as an initiator for the copolymerization of pentafluorophenyl methacrylate and hexyl methacrylate. Then DASA was grafted on the hydrophobic blocks. The resulting amphiphilic copolymer was self-assembled into polymersomes (30-100 nm). HRP was loaded in the polymersomes to form photoresponsive nanoreactors. Thanks to DASA switching, permeability of polymersome membranes were controlled under visible light irradiation $(630 \mathrm{~nm}$ and $525 \mathrm{~nm}$ ) thus enabling the control of the nanoreactors.

\subsubsection{Dimerization and dimer cleavage}

In a context of delivery application, dimerization processes have been proposed to form gels or stabilize particles before release [52] [103]. To induce the release of a loaded drug, the gel or particles are irradiated, uncrosslinked and, as a consequence, lose their stiffness or structural integrity [104] [105] [106] [107]. Three molecules are used as dimers for such application: coumarin [104] [105], cinnamate [106], and anthracene [107]. However, such release mechanisms by dimer cleavage is not really adapted for drug delivery applications in vivo. Indeed, UV exposure typically leading to hydrogel scission is too long for cell viability, reactions are not complete with side reactions and the process is not resistant to fatigue.

\section{Different drug release mechanisms}

In the previous part, the effect of light at the molecular level was discussed, together with the major mechanisms of action and their limitations. We now shift our focus to the effect of light activation when photoactive substances are incorporated into drug delivery systems. One can highlight two major mechanisms to induce and control drug release profiles: (1) a complete destabilization of the carrier / particle leading to a complete drug release, or (2) an acceleration of the drug diffusion through the carrier (membrane permeation or gel shrinkage).

\subsection{Light-induced structural changes inducing drug carrier destabilization}

A relevant question at this stage is: how can drug release be induced through carrier disruption? To answer this question, the underlying mechanisms responsible for the stabilization of the carrier structure have to be understood. With this in mind, two 
different classes of drug carrier can be proposed: (1) particles which are stabilized thanks to hydrophilic / hydrophilic interactions (such as micelles or vesicles) and (2) gels that have a 3-dimensional structure that is maintained due to physical [108] [109] or chemical crosslinking [110] [111].

\subsubsection{Self-assembled nanoparticles}

Polymer nanoparticles such as micelles, worm-like micelles or polymersomes are thermodynamically or dynamically stable self-assemblies as a result of copolymer amphiphilic properties. Indeed, in order to minimize the interaction of the hydrophobic blocks with water, block copolymers assemble into well-defined structures. Micelles and worm-like micelles are nanoparticles that are composed of a hydrophobic core surrounded by a hydrophilic shell. The difference between these two structures is the shape: micelles are spherical nanoparticles and worm-like micelles are cylindrical and elongated nanoparticles. Polymersomes are small artificial vesicles enclosing an aqueous solution separated by a bilayer membrane made of amphiphilic block copolymers. A key property to obtain and stabilize these structures is the hydrophobic to hydrophilic ratio of the two blocks [112]. Depending on this ratio, different structures or no specific structure can be obtained. As an interesting example, a hydrophilic block poly(glycerol monomethacrylate) (PGMA) was used as a chain transfer agent to polymerize in water 2-hydroxypropylmethacrylate (HPMA), a hydrophilic monomer [113], resulting in the formation of a well-defined amphiphilic diblock copolymer. During the HPMA polymerization, the hydrophobic ratio of the diblock increased leading the formation of different structures over time. The amphiphilic block starts to form micelles then worms and finally vesicles over time, depending on the ratio. This example shows how important the hydrophobic to hydrophilic ratio is in defining the structures formed. As a consequence, inducing a change in this equilibrium can destabilize the structure.

There are two ways to change the balance between the two chains of the amphiphilic polymer, either by modifying the hydrophobic to hydrophilic ratio [91] [94] or by separating the two blocks [114]. As far as we know, only one example of block separation has been reported for cargo delivery [114]. In this study poly $(\gamma-$ methyl- $\varepsilon-$ caprolactone) is used as the hydrophobic block and poly(acrylic acid) (PAA) as the hydrophilic block with a photo-cleavable o-nitrobenzyl (ONB) inserted between the two blocks. The amphiphilic block can self-assemble into polymersomes and micelles with a hydrodynamic radius of $83 \mathrm{~nm}$ and $31 \mathrm{~nm}$, respectively. After UV irradiation (365 nm), the two structures were disrupted and aggregates were formed. The second way to disrupt particles is to play with the change in the hydrophobic balance. The conversion of the hydrophobic blocks into hydrophilic blocks is the most popular mechanism found in the literature. This change is induced at the molecular level by cleavage, switching hydrophilic / hydrophobic properties, or azobenzene isomerization. As an example for cleavage-inducing destabilization, Jiang et al. synthesized amphiphilic copolymers of poly(ethylene oxide) and poly(methacrylate) 
bearing a hydrophobic pyrene moiety in the side group (PEO- $b$-PPy) [74]. These diblocks were able to self-assemble into micelles with an average diameter of $15 \mathrm{~nm}$. Under UV irradiation, the pyrene ester moiety is cleaved, and the hydrophobic PPy is converted into hydrophilic poly(methacrylic acid). Thus, after irradiation of the micelle solution for $15 \mathrm{~min}$ with UV (365 nm), the micelles were destabilized and the Nile red previously loaded was released (Fig. 9).
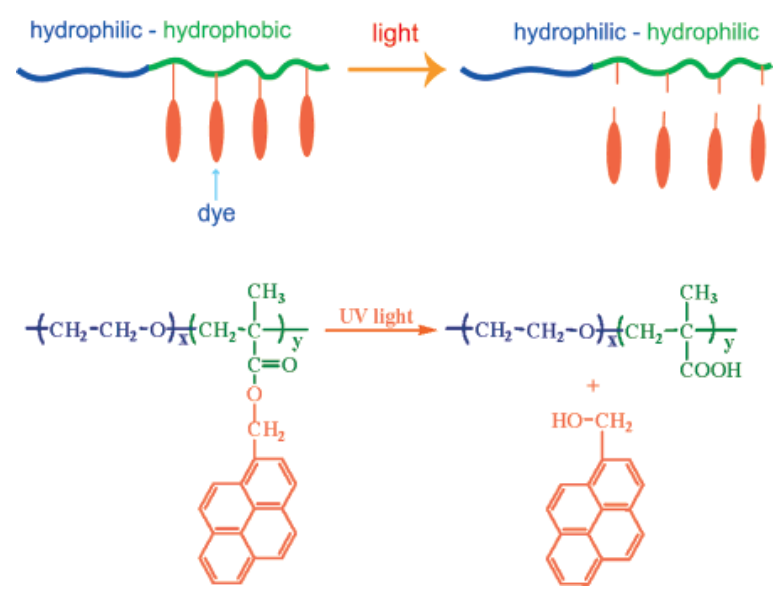

Fig. 9 Release of a hydrophobic group induced particle by destabilization [74] [Reprinted with permission from J. Jiang, X. Tong, Y. Zhao, A New Design for Light-Breakable Polymer Micelles, J. Am. Chem. Soc. 127 (2005) 8290-8291. Copyright (2005) American Chemical Society.]

Equally, an amphiphilic block of poly(benzyl carbamate) for the hydrophobic part and poly(N,N-dimethylacrylamide) as the hydrophilic part (PBC-b-PDMA) was employed. PBC exhibits, after chain-end cleavage, head to tail cascade depolymerization, which is also called self-immolation [53]. The hydrophobic block depolymerization induces a huge change in the hydrophilic to hydrophobic ratio of the copolymer. In this example the end chains are photocleavable thanks to perylen-3-yl (visible-light sensitive : $420 \mathrm{~nm}$ ) and 2-nitrobenzylbenzyl (UV sensitive : $365 \mathrm{~nm}$ ) groups. The PBC-b-PDMA can also self-assemble into vesicles with hydrodynamic diameters of around $250 \mathrm{~nm}$. The combination of all these properties results in light-sensitive self-immolative polymersomes. As a consequence, after irradiation at the appropriate wavelength (depending on the light-sensitive moiety) the chain end is cleaved inducing depolymerization, polymersome destabilization and Doxorubicin release, which was previously encapsulated.

The two previous examples illustrate how photo-cleavage can destabilized selfassembled particles.

Switching molecular hydrophobicity is a popular way to destabilize nanostructures by changing the hydrophilic to hydrophobic ratio. To this end, molecules presented in 2.2.2.2 have been widely used such as spiropyran [19] [96] [115] [116], DNQ [91] [92] [95] [99] and DASA (Fig. 11) [93][94]. 
As an example with spiropyran, this hydrophobic light-sensitive molecule was grafted with a hydrophilic, hyperbranched polyglycerol (SP-hb-PG) resulting in an amphiphilic polymer (Fig. 10) [96]. Moreover, this polymer can self-assemble into well-defined micelles with an average hydrodynamic diameter of $30 \mathrm{~nm}$. When these nanoparticles were irradiated with UV light $(365 \mathrm{~nm})$, the neutral hydrophobic spiropyran was isomerized into the hydrophilic zwitterionic merocyanine changing the hydrophilic ratio. As a consequence, micelles were destabilized and released pyrene, a hydrophobic test molecule.

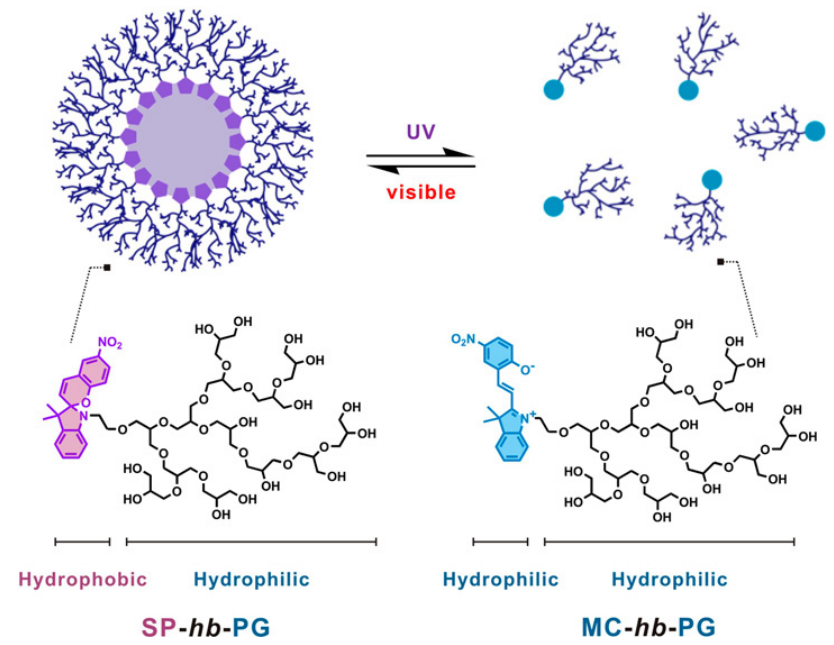

Fig. 10 Spiropyran hydrophilicity switch inducing particles destabilization [96] [Reprinted with permission from S.

Son, E. Shin, B.-S. Kim, Light-Responsive Micelles of Spiropyran Initiated Hyperbranched Polyglycerol for Smart Drug Delivery, Biomacromolecules. 15 (2014) 628-634. Copyright (2014) American Chemical Society.]

Wang et al. used DNQ as light sensitive moiety to disrupted particles [92]. In this study a diblock of poly(ethylene glycol)-block-poly(dimethylaminoethyl methacrylate). (PEG-b-PDMAEMA) was synthesized by ATRP. In addition, DNQ was grafted on the PDMAEMA block making it hydrophobic. As a result, an amphiphilic diblock (PEG-b-PDMAEMA@DNQ) was obtained. This diblock can self-assemble into micelles with an average diameter of $150 \mathrm{~nm}$. Irradiation by UV or NIR (365 nm or $808 \mathrm{~nm}$ ) leads DNQ to undergo a Wolf rearrangement and conversion of the hydrophobic block into a hydrophilic block. As a consequence of the hydrophilic ratio change, micelles are disrupted and $60 \%$ of coumarin 102 , chosen as a hydrophobic model drug, was released at $\mathrm{pH}=7$.

As an example that uses DASA, two hydrophobic n-heptane groups were grafted on DASA and a PEG at the other molecular extremity resulting in amphiphilic blocks that can self-assemble into micelles [94]. Irradiation with visible light (530 nm to $570 \mathrm{~nm}$ ) leads to the DASA conversion into its zwitterionic form, changes the copolymer hydrophilic ratio and releases Nile red or Paclitaxel, which is used to treat tumors, in cancerous cells.

These examples showed three light sensitive hydrophobicity switch molecules, which change the hydrophobic ratio of amphiphilic copolymers and disrupted selfassembled structures. 


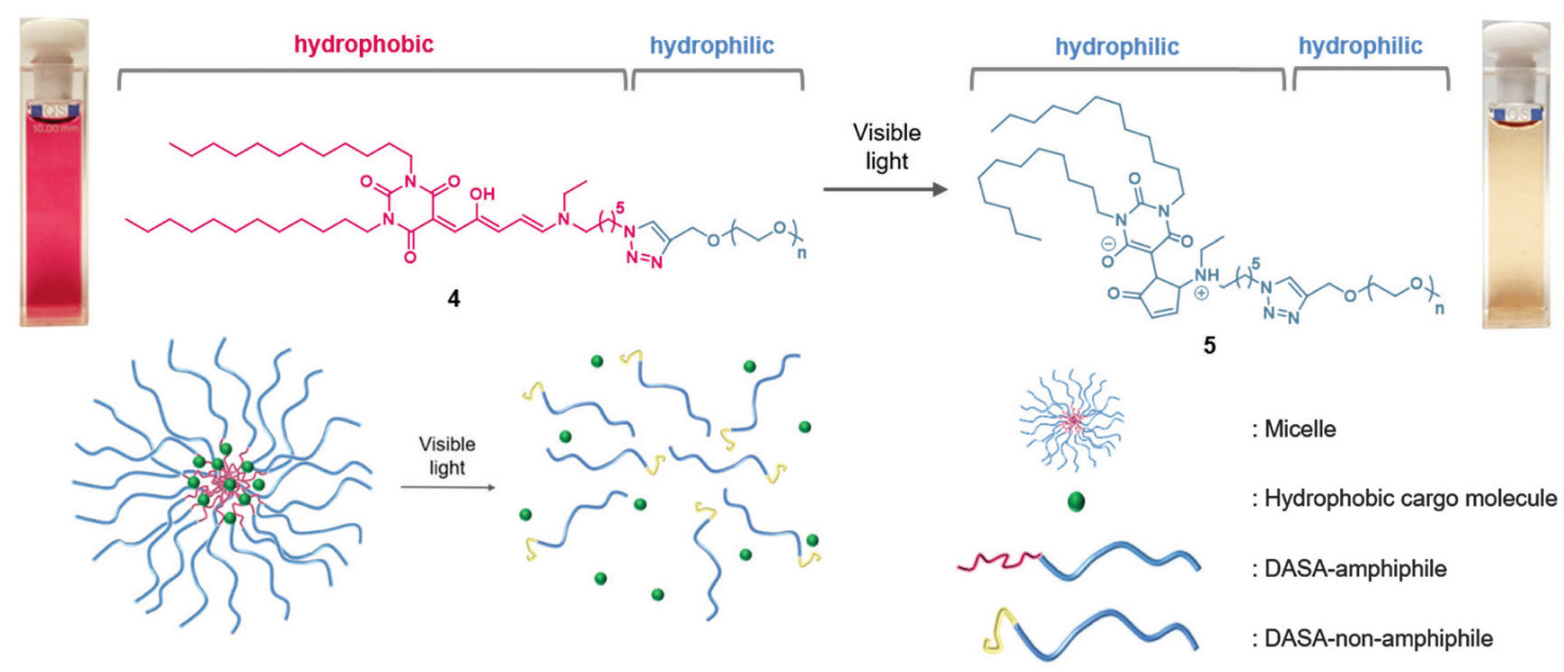

Fig. 11 DASA hydrophilicity switch inducing particles destabilization [94] [Reproduced from S.O. Poelma, S.S. Oh, S. Helmy, A.S. Knight, G.L. Burnett, H.T. Soh, C.J. Hawker, J.R. de Alaniz, Controlled drug release to cancer cells from modular one-photon visible light-responsive micellar system, Chem. Commun. 52 (2016) 10525-10528 with permission of The Royal Society of Chemistry.]

The azobenzene isomerization can also induce particle disruption. Azobenzene isomers ( $Z$ and $E$ ) have different dipole moments and as a consequence the cisisomer is more hydrophilic than trans-isomer [117]. When azobenzene is integrated in polymer chains this hydrophobicity difference is enough to disrupt micelles or vesicles. As an example, amphiphilic block copolymers of poly(methacrylate) containing-azobenzene as the hydrophobic block and as the hydrophilic block a statistical copolymer of poly(acrylic acid) and poly(tert-butyl acrylate) (PAzoMA- $b$ PAA-PtBA)), were synthesized [118]. Depending on the block sizes, copolymers selfassembled into micelles (15 nm with PAzoMA $\left.{ }_{31}-b-\mathrm{PAA}_{33}-\mathrm{PtBA}_{19}\right)$ or vesicles (100$300 \mathrm{~nm}$ with PAzoMA ${ }_{74}-b-\mathrm{PAA}_{22}-\mathrm{PtBA}_{46}$ ). Under UV light ( $360 \mathrm{~nm}, 10 \mathrm{~min}$ ) azobenzene grafted on the hydrophobic polymer chain isomerized into its cis- form leading to micelle or vesicle disruption. Azobenzene isomerization is reversible and the copolymer solution previously irradiated self-assembled again into micelle or vesicles under visible light $(440 \mathrm{~nm}, 3 \mathrm{~min})$. Having been reset, this process can be repeated several times.

\subsubsection{Giant polymersomes}

Mechanisms involved in the release of species from giant polymersomes (whose size is $>1 \mu \mathrm{m}$ ) are somewhat different than nano-polymersomes. Three examples, that may be considered particularly relevant and original, are presented here. Mabrouk et al. produced light sensitive asymmetric polymersomes that burst under a light stimulus [86]. The property differences between the inner and outer leaflet under UV irradiation induces membrane frustration and destabilization. To produce these micrometric asymmetric polymersomes, the emulsion-centrifugation [119] technique was used. This method consists in stabilizing water emulsion droplets in an organic solvent (in this case toluene) thanks to an amphiphilic block copolymer. After 
centrifugation, emulsion droplets crossed the interface of a water / organic phase, also stabilized by an amphiphilic block copolymer. With this technique, two different copolymers can be used for the emulsion and the interface in order to generate an asymmetric membrane. While the emulsion droplets cross the interface from the organic phase to the water phase, a second leaflet of copolymer is added around the droplets, thus forming polymersomes. In this study the two copolymers used were poly(ethylene glycol)- $b$-polybutadiene (PEG- $b$-PBD) and a liquid crystalline copolymer PEG-b-poly(4-butyloxy-2'-(4-(methacryloyloxy)butoxy)-4'-(4butoxybenzoyloxy)azobenzene) (PEG-b-PMAazo444). The hydrophobic part consisted in a poly(methacrylate) bearing a modified azobenzene group. Without UV irradiation, azobenzene is in its thermodynamically stable trans- form and acts as a well-ordered liquid crystalline. Under UV irradiation azobenzene isomerizes into its cis- form. The leaflet containing PEG- $b$-PMAazo444 has a disordered state, its projected area increases and swells compared to the other leaflet. This induces a membrane tension increase and, consequently, in order to relax this tension, the membrane collapses and the polymersome bursts releasing its content.

In another study a ferritin protein, that stores and releases iron in cells, was encapsulated in PEG- $b$-PBD polymersomes (around $10 \mu \mathrm{m}$ ) and adsorbed in the inner leaflet of the membrane [27]. In addition, bis[(porphinato)zinc] $\left(\mathrm{PZn}_{2}\right)$ was embedded in the polymersome membrane. This chromophore absorbs in the near UV, visible, and NIR light. The system was irradiated at $488 \mathrm{~nm}, 543 \mathrm{~nm}$ or $633 \mathrm{~nm}$ where the $\mathrm{PZn}_{2}$ absorbs strongly. As a consequence, morphological changes in vesicles such as budding of smaller vesicles and vesicle rupture was induced. Indeed, the synergy of the ferritin and the $\mathrm{PZn}_{2}$ converted light energy into heat energy that generated differential rigidification in the membrane, stressed the membrane and ruptured or budded vesicles. To demonstrate photorelease of a small model drug, biocytin was encapsulated and 25 - 50\% was released after irradiation.

Recently, Peyret et al. loaded three photocleavable molecules in giant polymersomes, namely calcein, methylene blue and a coumarin derivative using an emulsion-centrifugation method [120]. These molecules served to rapidly increase the osmotic pressure inside the polymersome lumen by increasing the number of species after photocleavage under irradiation. Calcein polymersomes, methylene blue polymersomes and coumarin derivative polymersomes were, respectively, irradiated at $488 \mathrm{~nm}, 633 \mathrm{~nm}$ and $405 \mathrm{~nm}$ in a confocal microscope, which induced fast polymersome bursting, in the range of milliseconds to seconds. Indeed, as polymersomes are impermeable to water, rapid osmotic pressure change cannot be compensated, which results in membrane stress and consequently polymersome rupture. Polymersomes encapsulating the different aforementioned dyes, rendering them sensitive to different irradiation wavelengths, were burst independently with high specificity and temporal precision in the same medium. These light-sensitive polymersomes were further able to release nano-polymersomes and nanoliposomes. This approach can obviously be used to release any kind of loaded cargoes in a very precise manner. 


\subsubsection{Gels}

Gels are effective drug delivery systems, having high loading capacity, stability and often good biocompatibility when inert hydrophilic segments are used. Gels are generally robust, especially in the case of chemically crosslinked 3D gels, can be formulated into large pieces (macro gels) that can be implanted or small nanoparticles (nanogels) that can circulate in the blood stream [121]. Nanogels can also be combined with inorganic nanoparticles to form a shell [20]. Gels are either chemically [110] [111] or physically crosslinked [108][109]. Physical gels are reversible gels that are crosslinked via molecular interactions or secondary forces such as van der Waals, ionic or hydrophobic interactions. As an example of a physical gel, a triblock ABA was prepared with hydrophilic PEG as the B block and hydrophobic photolabile poly([6-bromo-7-hydroxycoumarin-4-yl]methyl methacrylate) (PBHCMM) as a the A block resulting in PBHCMM- $b$-PEG- $b$-PBHCMM [108]. The ABA triblock was used to prepare a macro hydrogel. As shown in Fig. 12, the hydrophobic interactions of the A blocks are responsible for the formation of a 3D network. In another example, a polysaccharide, the polymaltotriose (or pullulan), bearing a hydrophobic cholesteryl group was used to form nanogel particles with a hydrodynamic diameter of $18 \mathrm{~nm}$ [109]. The hydrophobic moiety acts as the physical link to create the polymer network and the gel. Chemical gels or permanent gels are held with covalent bonds. An example of chemical gel is presented in Fig. 14 : a 4armed PEG tetra coumarin bearing an azido group and a 4-armed PEG tetra-alkyne were synthesized [110]. The two 4-armed polymers in water undergo a Huisgen click reaction using the azido group, the alkyne and copper as a catalyst, creating covalent junctions resulting in a macro hydrogel. To induce release of the drug previously loaded in the hydrogel, the method widely used is to dissolve and disintegrate hydrogels by rupture of the chemical or physical junctions responsible for the network.

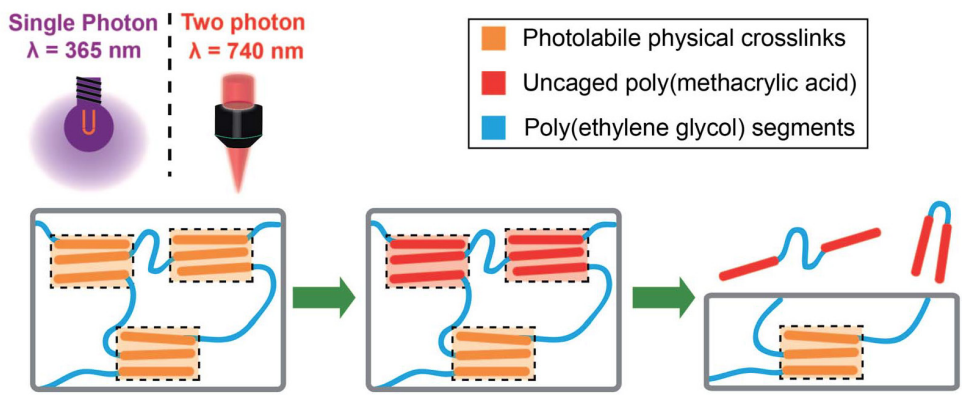

Fig. 12 Example of a light sensitive physical gel [108] [Reproduced from C. Zhu, C.J. Bettinger, Light-induced remodeling of physically crosslinked hydrogels using near-IR wavelengths, J. Mater. Chem. B. 2 (2014) 16131618 with permission of The Royal Society of Chemistry].

For physical gels, destabilization can be induced by cleavage of the hydrophobic moiety responsible for the physical junctions. As an example, we cited above the case of the triblock PBHCMM- $b-P E G-b-P B H C M M$ [108]. Each end-chain 
of the triblock bears one cleavable hydrophobic coumarin. Under UV or NIR irradiation (365 nm or $740 \mathrm{~nm}$ ) the coumarin is uncaged and the hydrophobic PBHCMM transforms into hydrophilic poly(methacrylic acid) (PMMA), thus destabilizing the hydrogel ([109]). In another interesting example cited above, the hydrophobic cholesteryl group was covalently-linked to the hydrophilic polysaccharide by a photocleavable ONB [109]. FITC-insulin was encapsulated in the nanogel. A film was formed with the FITC-insulin nanogel particles and under UV irradiation (365 nm, $50 \mathrm{~min}$ ) the cholesteryl group was cleaved. Consequently, the physical bonds are ruptured, the nanogel film is disrupted and FITC-insulin is released.

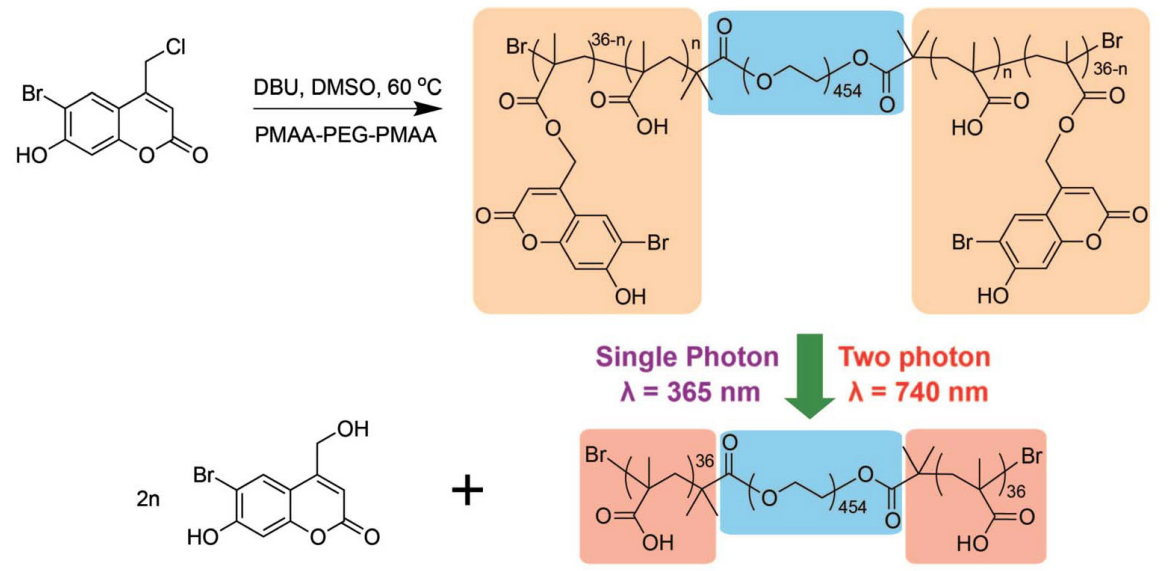

Fig. 13 Photocleavage of PBHCMM-b-PEG-b-PBHCMM inducing destabilization of the physical hydrogel [108] [Reproduced from C. Zhu, C.J. Bettinger, Light-induced remodeling of physically crosslinked hydrogels using near-IR wavelengths, J. Mater. Chem. B. 2 (2014) 1613-1618 with permission of The Royal Society of Chemistry].

In the case of chemical gels, destabilization occurs via covalent bond rupture (Fig. 14). These bonds can be cleaved using of coumarin [110] [122], ONB [20] [111] or a diazo linker [78].

In an aforementioned study, 4-armed PEG bearing photo-cleavable coumarin moieties were crosslinked [110]. The macro hydrogel was irradiated with UV light (365 nm or $405 \mathrm{~nm}$ ), inducing coumarin cleavage and chemical junction rupture, resulting in the hydrogel destabilization (Fig. 14). Coumarin was further used as a crosslinker to form a polystyrene microgel by emulsion polymerization [122]. The final microgel particles were between $100 \mathrm{~nm}$ and $200 \mathrm{~nm}$ and under UV light (365 nm) the coumarin was cleaved, the hydrogel was degraded and Nile red was released. 


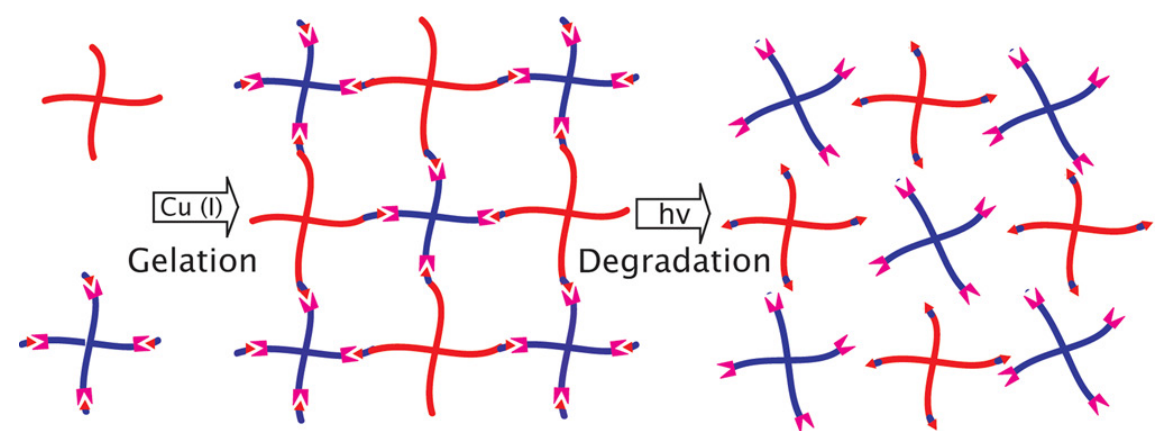

Fig. 14 Example of the destabilization of a chemically crosslinked gel [110] [Reprinted with permission from M.A. Azagarsamy, D.D. McKinnon, D.L. Alge, K.S. Anseth, Coumarin-Based Photodegradable Hydrogel: Design, Synthesis, Gelation, and Degradation Kinetics, ACS Macro Lett. 3 (2014) 515-519 Copyright (2014) American Chemical Society.]

In a last recent and very elegant example concerning light-sensitive degradable hydrogels, Badeau et al. reported gel junctions that behave according to Boolean logic. Three cleavable molecules which respond to different stimuli were chosen : one sensitive to light (ONB), one sensitive to reduction (disulfur bond) and one sensitive to an enzyme (metalloproteinase). By connecting cleavable groups in series or in parallel (Fig. 15), they managed to tune the junctions sensitivity with AND/OR connectors, then crosslinked with the junction 4-armed PEG and formed a hydrogel with the same sensitivity. For example (Fig. 15), they synthesized a hydrogel that undergoes degradation after light irradiation (A) AND (reduction OR presence of the enzyme) [123].
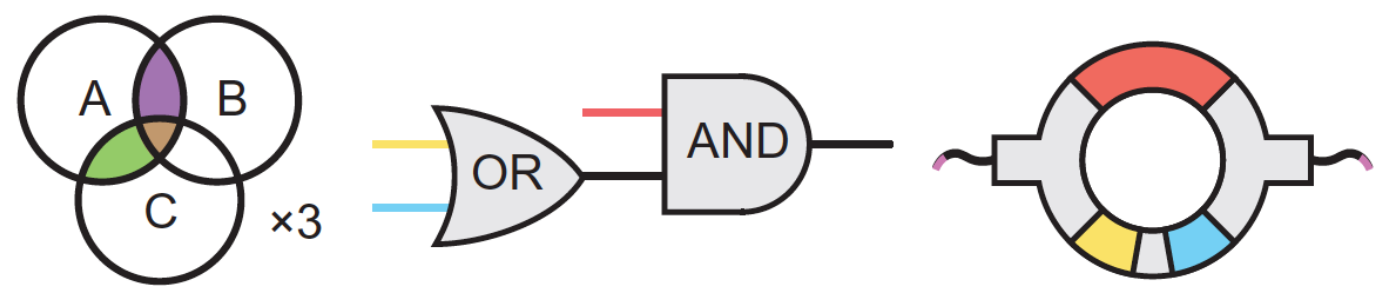

Fig. 15 Logic connector which can destabilize gel with AND and OR stimuli [123] [Reprinted by permission from Springer Nature: Nature Chemistry, B.A. Badeau, M.P. Comerford, C.K. Arakawa, J.A. Shadish, C.A. DeForest, Engineered modular biomaterial logic gates for environmentally triggered therapeutic deliver, copyright (2018)]

\subsection{Light-induced increase of diffusion speed (Heat generation / permeability modification)}

In contrast to the previous section, the systems described here can release their content while maintaining their structure. Drug release is induced by changing the diffusion properties from the carrier.

A simple way to increase the diffusion rate is based on temperature. As seen in part 1, nanoheaters increase system temperature due to light radiation. To have a light sensitive drug release system, nanoheaters can be combined with thermosensitive carriers. There are a wide variety of nanoheaters and a wide variety of thermosensitive polymers [124] [125] and consequently there are tremendous 
possibilities to combine them both to design of light sensitive drug delivery systems with appropriate properties.

In this review, we will focus on a few demonstrative examples that we consider the most relevant. Two different approaches are presented here, based on (1) polymers with a lower critical solution temperature (LCST) and (2) hydrophobic polymers that undergo phase transition.

\subsubsection{Polymer / Gel shrinkage (solubility change)}

The LCST is the temperature above which the component becomes insoluble in a given solvent. In water, when the temperature is increased above this critical temperature, the polymer chains pass from a hydrophilic swollen state to hydrophobic shrunken state [124]. For drug delivery applications active substances are trapped in the carrier swollen state. When light is activated the nanoheater increases the temperature above the LCST and the carrier shrinks. As a result of the resulting volume reduction, the drug is released [41]. To have an efficient delivery system the polymer LCST should be a slightly larger than the body temperature $\left(37^{\circ} \mathrm{C}\right)$. The LCST can depend on the molar mass, component concentration or $\mathrm{pH}$ [124]. The most important polymers used for thermally sensitive drug delivery systems are poly( $\mathrm{N}$-isopropylacrylamide) (pNIPAAM), copolymers of poly(ethylene oxide), poly(propylene oxide) (often referred as pluronics or jeffamine [126]) and elastin-like polypeptides (ELP [127]). However, mainly pNIPAAM combined with nanoheaters has been used for light sensitive drug delivery systems. pNIPAAM has interesting properties: its LCST only slightly depends on its molar mass, $\mathrm{pH}$ or concentration [128]. Its LCST that is around the body temperature $\left(30^{\circ} \mathrm{C}\right.$ to $\left.35^{\circ} \mathrm{C}\right)$ has to be tuned to be higher. With this in mind, interesting gels based on the copolymerization of NIPAAM with methacrylic acid (MAA) and N,N'-methylene-bisacrylamide (MBA) (crosslinker), were obtained with an appropriate LCST. These gels were polymerized directly around nanoheaters $\left(\mathrm{Cu}_{1.75} \mathrm{~S}\right.$ nanocrystals $)$ and when the system was irradiated with NIR $(808 \mathrm{~nm})$ it efficiently released doxorubicin that was previously trapped in the polymer blend (Fig. 16) [13]. 
In another study, NIPAAM and acrylamide (AAM) were copolymerized to tune the LCST. The obtained copolymers were covalently anchored on gold nanocages (Fig. 3 ). These cages have pores that are obstructed when the polymer is in its swollen state (below LCST). After irradiation in the NIR, the gold nanocages locally heated the polymer chains that collapsed and shrank. The pores were no longer obstructed leading to doxorubicin release [14].

Temperature change is not the only way to induce polymers or gels shrinkage. Hydrophobic transition thanks to cleavage or the introduction of hydrophobicity switched molecules can also provoke a volume transition in materials. In this context, a hydrophobic coumarin moiety was grafted on a hyaluronic acid (HA) hydrogel (Fig. 17). This gel formed well-defined nanoparticles that could efficiently load doxorubicin due to the hydrophobic interactions with coumarin. After UV irradiation (315-400 nm) the hydrophobic coumarin derivatives were cleaved, making the HA nanogels more hydrophilic, thus allowing its swelling and the release of doxorubicin [66].

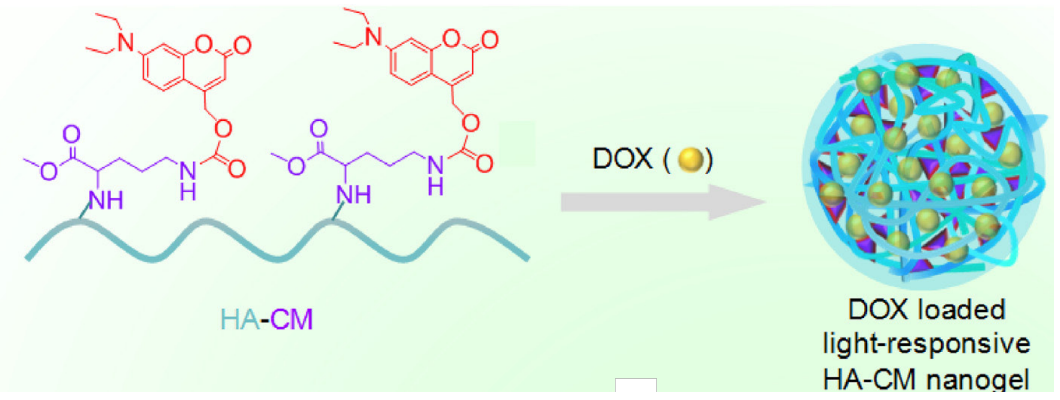

Fig. 17 Coumarin release induces gel swelling [66] [Reprinted from "Hang, Y. Zou, Y. Zhong, Z. Zhong, F. Meng, NIR and UV-responsive degradable hyaluronic acid nanogels for CD44-targeted and remotely triggered intracellular doxorubicin delivery, Colloids Surf. B Biointerfaces. 158 (2017) 547-555" Copyright 2017 with permission of Elsevier]

In another study the transition from the hydrophobic spiropyran form to the hydrophilic zwitterionic merocyanine form was also shown to shrink micelle particles (Fig. 18). This shrinkage allows a faster release of drugs and a deeper tissue penetration [90]. Spiropyran (SP) linked to a lipidic chain (thus fully hydrophobic) (SP-C9) and 1,2-distearoyl-sn-glycero-3-phosphoethanolamine-poly(ethylene glycol) (DSPE- PEG) were nanoprecipitated together resulting in hybrid micelle formation. These nanoparticles had a very low dispersity (PDI $=0.03$ ) with a hydrodynamic diameter of $150 \mathrm{~nm}$. Moreover, they were irradiated under UV light (365 nm) and the hydrophobic chain of SP-C9 became amphiphilic. Consequently, the micelles shrunk to a size of $47 \mathrm{~nm}(\mathrm{PDI}=0.05)$. Different drugs were successfully loaded in these micelles: rhodamine $\mathrm{B}$, coumarin 6 , cyanine 5 (Cy5), paclitaxel, docetaxel, proparacaine, and doxorubicin, up to $10 \mathrm{wt} \%$. Particle shrinkage induced release of encapsulated drugs. Another major advantage of such particle shrinkage was demonstrated in this study: after UV irradiation, diffusion of the nanoparticles into collagen tissues increased due to their reduced sizes. Finally, the authors used the micelles to successfully deliver Cy5 into porcine cornea thanks to their reduced sizes after irradiation. 


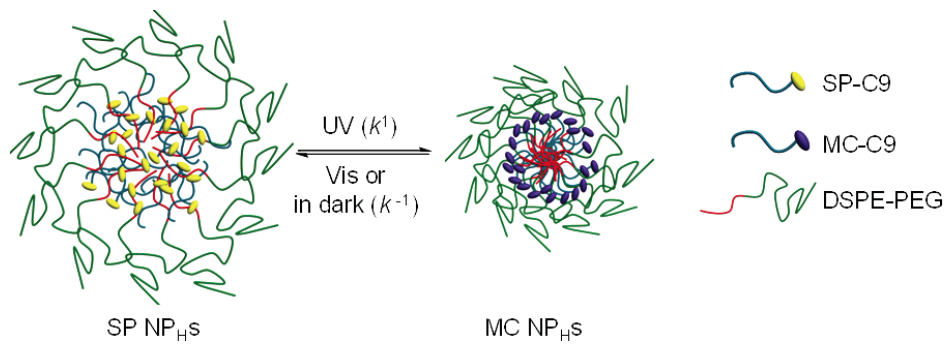

Fig. 18 Spiropyran hydrophilicity switch induces nanoparticle shrinkage [90] [Reprinted from "R. Tong, H.D. Hemmati, R. Langer, D.S. Kohane, Photoswitchable Nanoparticles for Triggered Tissue Penetration and Drug Delivery, J. Am. Chem. Soc. 134 (2012) 8848-8855 Copyright (2014" with permission of American Chemical Society]

\subsubsection{Glass transition and melting induced release}

Two intrinsic polymer properties are mainly controlling the drug diffusion rate in a self-assembled polymer system: their glass transition ( $\mathrm{Tg}$ ) and melting (Tm) temperatures. The glass transition temperature is the temperature at which the polymer goes from an amorphous state (hard and brittle) to a rubbery or liquid viscous one. Melting temperature is the temperature at which the crystalline part of a polymer becomes liquid. Polymers with a melting or glass transition temperature just above $37^{\circ} \mathrm{C}$ are particularly interesting for drug delivery applications. These polymers can be associated with nanoheaters and the heat generated during the irradiation can increase the diffusion rate.

Poly(lactic-co-glycolic acid) (PLGA) is an amorphous polymer with a Tg above $37^{\circ} \mathrm{C}$ [129]. Above its $\mathrm{Tg}$ the polymer is softened and more permeable to drugs. Moreover, this FDA-approved polymer degrades by hydrolysis and the organism is not adversely affected by the decomposition products [130]. An interesting example took advantage of the low Tg of PLGA. PLGA nanoparticles containing doxorubicin were formed thanks to nanoemulsion technique [15]. Gold nanospheres were fixed around the PLGA nanoparticles, an additional gold layer was added, resulting in PLGA loaded particles surrounded by a gold nanoshell with diameter of $80 \mathrm{~nm}$ (Fig. 19). Anti-EGFR(epithermal growth factor receptor) antibody (Cetuximab) was grafted on the gold nanoshell in order to target epithelial cancer cells. Irradiation with NIR laser $(820 \mathrm{~nm})$ increased the gold nanoshell temperature. The heat produced was transferred to the polymer matrix and rose its temperature above its Tg. As a consequence, the polymer matrix was softened and doxorubicin diffusion was accelerated compared to non-irradiated nanoparticles. These PLGA loaded doxorubicin nanoparticles with a gold nanoshell and antibody were then tested on cells. Nanoparticles showed a significantly greater affinity with cells expressing a high level of EGFR. In addition, irradiated cells with nanoparticles presented a $69.8 \%$ lower cell viability compared to non-irradiated cells with nanoparticles, showing the system efficacy. Another study used the same principle using PLGA loaded doxorubicin nanoparticles with a gold nanoshell and antibody [131]. In addition, these nanoparticles were tested on mice showing an active targeting of the tumor. As a 
result, the tumor was completely destroyed thanks to both heat and delivered doxorubicin.

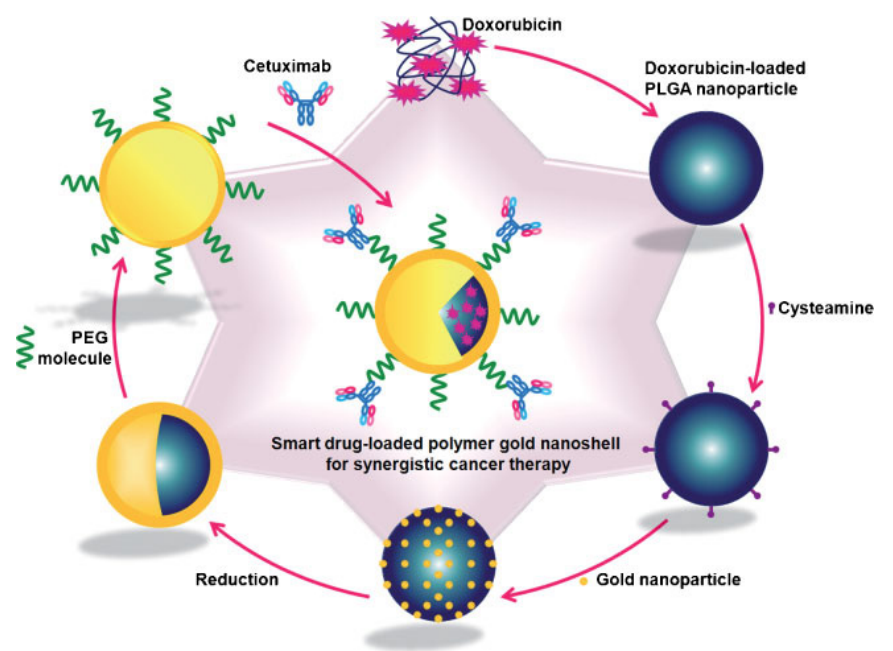

Fig. 19 PLGA surrounded with gold nanocarriers and NIR induced doxorubicin release [15] [Reprinted from " J. Yang, J. Lee, J. Kang, S.J. Oh, H.-J. Ko, J.-H. Son, K. Lee, J.-S. Suh, Y.-M. Huh, S. Haam, Smart Drug-Loaded Polymer Gold Nanoshells for Systemic and Localized Therapy of Human Epithelial Cancer, Adv. Mater. 21 (2009) 4339-4342" with permission of John Wiley and sons]

Agarose gel was also used to release protein with visible light due to heating from gold nanospheres incorporated inside the gel (Fig. 20) [16]. In this example, gold nanospheres, with a diameter of $70 \mathrm{~nm}$, were incorporated in a macroscopic agarose hydrogel loaded with bevacizumab, a protein used for its anti-angiogenic properties. Under visible light (400-500 $\mathrm{nm}$ ) the gold nanospheres convert light energy into heat energy and transfer it to the hydrogel. The temperature increase induced a softening effect of the agarose gel and as a consequence increased the diffusion rate of the therapeutic protein. The softening effect is reversible, thus the system can be turned on and off for several cycles. This system was injected in bovine eyes and after visible light irradiation protein release was detected.

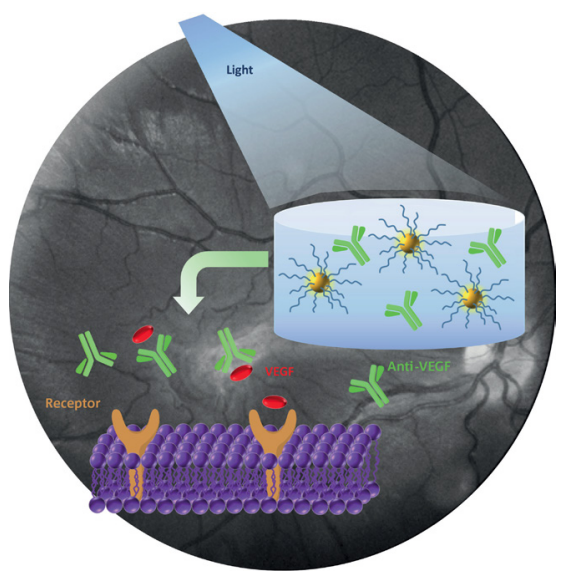

Fig. 20 Agarose gel releases protein under light stimulus [16]. [Reprinted from "Photo-Modulated Therapeutic Protein Release from a Hydrogel Depot Using Visible Light - Basuki - 2016 - Angewandte Chemie " with permission of John Wiley and sons ] 
Melting temperature is the second intrinsic parameter that can be used to increase the diffusion rate. In this case, two polymers are particularly well-adapted due to their low melting temperature and biocompatibility: PTMC and PCL, with melting temperatures of $37^{\circ} \mathrm{C}$ and $52^{\circ} \mathrm{C}$, respectively [124]. To the best of our knowledge these polymers have not been used for light sensitive systems, even if a similar concept consisting in a local heating induced by magnetic hyperthermia has shown selective release of doxorubicin and specific toxicity induction in PTMC based polymersomes [132].

\subsection{Pore blocking / unblocking}

One way to trigger release from nano-carriers is through pores, which offers a way for cells to release species [133]. This can be highly selective [134] and triggered with different stimuli, like light [88]. Forming or unblocking pores can be achieved via cleavage, isomerization, hydrophobicity switched molecules or nanoheaters.

In an example using isomerization for pore unblocking, azobenzene was used to open pore proteins in a cell membrane (Fig. 21). In this case the final study purpose was not for drug delivery application but rather to control cellular chemistry. This example is detailed being an interesting proof-of-concept. An amino acidsensitive membrane channel that opens in the presence of glutamate was used. The goal of this study is to be able to open the channel on demand thanks to light and to have a precise control of cellular chemistry in space and time. To do so, tetra-ortho substiuated azobenzene was used because of its stability, red-light switching and its cis- to trans- thermal relaxation presented a relative long half-life at $37^{\circ} \mathrm{C}: 3.5 \mathrm{~h}$. This azobenzene derivative was modified to bear one glutamate molecule on one extremity. The other extremity was used to graft the azobenzene derivative on the protein channel and two azobenzene derivatives were grafted on the protein channel. Without light, the azobenzene is in its trans- isomer form, glutamate is too far from the receptor to be activated and the channel remains closed. After UV or red-light irradiation the trans- isomer switched to the cis- isomer the glutamate gets closer to the receptor and activates it, resulting in its opening. Several wavelengths have been tested from $340 \mathrm{~nm}$ to $640 \mathrm{~nm}$, and the systems shows responses up to $640 \mathrm{~nm}$. The switching was reversible with blue light $(440 \mathrm{~nm})$ [88].

LiGluR

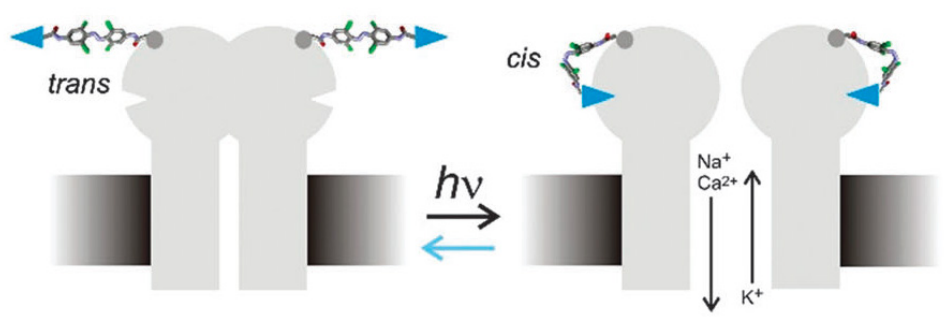

Fig. 21 Light induced azobenzene isomerization and pore opening [88] [Reprinted from "A. Rullo, A. Reiner, A. Reiter, D. Trauner, E.Y. Isacoff, G.A. Woolley, Long wavelength optical control of glutamate receptor ion channels using a tetra-ortho-substituted azobenzene derivative, Chem. Commun. 50 (2014) 14613-14615" Creative Commons Attribution 3.0 Unported Licence ] 
In another example using azobenzene isomerization, mesoporous silica nanoparticles (MSNs) with length and width of $200 \mathrm{~nm}$ and $150 \mathrm{~nm}$, whose pores were blocked with azobenzene and cyclodextrin, were used. The azobenzene has 4methoxy substitutions to shift the isomerization wavelength to the red. Due to the azobenzene trans-form geometry, $\beta$-cyclodextrin can envelop this isomer and form a complex. The azobenzene was linked to pores, as a consequence, due to the presence of $\beta$-cyclodextrin and the resulting complex, pores were obstructed. An interesting property of this complex is that the cis- isomer of azobenzene can no longer form a complex with $\beta$-cyclodextrin, therefore under visible light $(625 \mathrm{~nm})$ the $\beta$-cyclodextrin is released from azobenzene cis- form and pores were unblocked. This mechanism allowed to release doxorubicin stuck inside the pores [89].

Besides isomerization, cleavage have been used to unblock pores for drug delivery applications. Ruthenium complexes $\left(\left[\mathrm{Ru}(\mathrm{bpy})_{2}\left(\mathrm{PPh}_{3}\right) \mathrm{Cl}\right] \mathrm{Cl}\right)$ were covalently linked to the MSN walls and obstructed the pores [70]. In this study, MSNs had diameters of 100-200 nm and their pores sizes were $2.2 \mathrm{~nm}$. In addition, the ruthenium complex measured about $1.5 \mathrm{~nm}$ and was able to obstruct the MSNs pores. Under visible light $(455 \mathrm{~nm})$ the ruthenium complex was cleaved, released, the pores were opened and sulforhodamine 101 dye was released.

Additionally, polymer LCST have been used to unblock pores. As an example, stimuli-responsive pNIPAAM-co-pAAM was used to obstruct pores of gold nanocage [14] (Fig. 3). Gold nanocage sizes were around $50 \mathrm{~nm}$ and their pores on the edges measured between $5 \mathrm{~nm}$ and $10 \mathrm{~nm}$. The pores were closed due to swollen pNIPAAM-co-pAAM covalently linked to the cage and the layer of copolymer at its swollen state had a thickness of $5 \mathrm{~nm}$ that was sufficient to obstruct the pores. As mentioned previously, pNIPAAM has a LCST around the body temperature. A Ti:sapphire laser was used to irradiate NIR on the gold nanocage-copolymer system. As a result, the gold nanocage increased the copolymer temperature, which consequently shrunk. The copolymer thickness was reduced, the pores were no longer obstructed and previously loaded doxorubicin was released. This system was shown to kill breast cancer cells.

An alternative to pore unblocking is to directly create pores in the drug carrier. To this end isomerization hydrophobicity / hydrophilicity switch molecules and nanoheaters have been used. As an example, azobenzene isomerization was used to induce pore formation. A cationic azobenzene derivative: 4-cholesterocarbonyl- 4'(N,N,N-triethylamine butyloxyl bromide) azobenzene and surfactant sodium dodecyl sulfate were self-assembled into unilamellar and multilamellar vesicles with an average hydrodynamic diameter of $250 \mathrm{~nm}$. The UV irradiation (365 nm) triggered azobenzene isomerization and increased the inter-lamellar spacing. This nanostructural changes in the membrane induced pores formation and doxorubicin release [135]. This system was tested on rat retinas and could efficiently release drug and maintain high level of drug concentration for 8 hours.

Equally spiropyran was used to create pores in the DDS. For self-assembled particles such as polymersomes, hydrophobic blocks prevent the drug from leaving 
the drug carriers. A slight change in the hydrophilic balance of the block can induce a permeability increase without disturbing the drug carriers. This permeability change can be induced by a molecular hydrophobicity switch like spiropyran. In one article, spiropyran was grafted on an amphiphilic block copolymer PEG-b-PMMA using carbamate linkage: PEG- $b$-PSPA (Fig. 22). This copolymer was self-assembled into well-defined vesicles $(70 \mathrm{~nm}$ or $450 \mathrm{~nm}$ depending on the block lengths and PDI<0.1). Irradiation with UV light (365 nm) induces switching of spiropyran incorporated in the vesicle membranes and conversion of the spiropyran into the hydrophilic merocyanine. This switch increased the membrane permeability without destabilizing it. The carbamate hydrogen bonding helped to maintain the structure, which was destabilized by a significant hydrophilicity change in the membrane. After irradiation, the vesicles released small hydrophilic molecules 2'-deoxy-5-fluorouridine an anticancer drugs [136]. Permeability change of the vesicle membrane was reversible under visible light $(530 \mathrm{~nm})$.

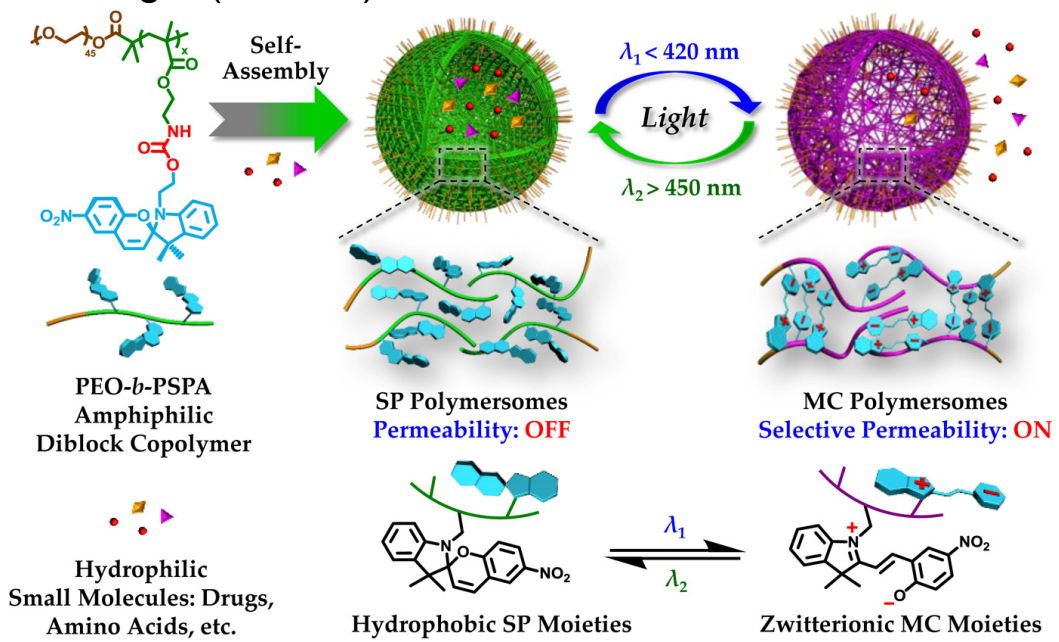

Fig. 22 PEG-b-PSPA self-assembled into light-sensitive polymersomes, whose permeability can be modulated under light irradiation using a spiropyran group [136]. [Reprinted from: X. Wang, J. Hu, G. Liu, J. Tian, H. Wang, M. Gong, S. Liu, Reversibly Switching Bilayer Permeability and Release Modules of Photochromic Polymersomes

Stabilized by Cooperative Noncovalent Interactions, J. Am. Chem. Soc. 137 (2015) 15262-15275, ACS AuthorChoice License]

Another group of particles made of gold sphere crosslinked together were used as a drug delivery systems [137]. Gold nanospheres $(10 \mathrm{~nm})$ were self-assembled and formed hollow spheres with an average hydrodynamic diameter of $65 \mathrm{~nm}$. Then the gold nanospheres were crosslinked with a dithiol-PEG in order to increase the stability in water. Visible or NIR light (532 $\mathrm{nm}$ and $790 \mathrm{~nm}$ ) irradiation induces a temperature increase of the gold nanospheres. As a consequence, the size of the nanogaps between the gold nanosphere increased from $2.5 \mathrm{~nm}$ to $4 \mathrm{~nm}$ while maintaining the hollow sphere structure thanks to the crosslinking. The difference of sizes with or without irradiation is enough to induce cargo leakage. The Rhodamine B dye and doxorubicin were loaded in the hollow spheres and under visible or NIR light, were released.

Besides spiropyran, DASA was used as a hydrophobicity switch molecule to increase permeability in polymersome membranes under visible light irradiation. In a very recent example PEG was used as a macro chain transfer with RAFT radical 
polymerization to extend the chain with the hydrophobic monomers pentafluorophenyl methacrylate (PFPMA) and hexyl methacrylate (HMA) both randomly distributed [102]. The backbone was then modified to graft on the PFPMA two different DASA moiety: Meldrum's acid based furan adduct (MELD) and a novel five-membered ring pyrazolone-based furan adduct. As a result two different amphiphilic block copolymer were synthesized and can self-assemble into vesicles between $30 \mathrm{~nm}$ and $200 \mathrm{~nm}$ depending on the copolymer used. MELD and PYRA polymersomes showed fast hydrophilic switching kinetics, membrane permeability increase and dye release under irradiation with respectively green light $\left(\lambda_{\mathrm{em}}=525\right.$ $\mathrm{nm})$ and red light $\left(\lambda_{\mathrm{em}}=630 \mathrm{~nm}\right)$. GOx and HRP enzymes were encapsulated separately in these polymersomes which are sensitive to two different wavelengths.. On mixing together the nanoreactors cascade reactions activated by red and green light at the same time could be performed.

\section{Conclusion}

The development of innovative drug delivery systems, able to control the appropriate therapeutic dose both spatially and temporally, is still very challenging and critical for treatment of many diseases. Long lasting delivery of molecular drugs and even biomacromolecules is rather well controlled, especially using biocompatible and biodegradable polymers. Indeed, many polymers and biopolymers have been designed over the past decades and are now widely used as biomaterials. In most cases, delivery is controlled by endogenous factors, such as the $\mathrm{pH}$ or the presence of specific enzymes or oxidant species, with a process of particle (mostly micro) degradation or erosion. However, when considering the use of very toxic drugs, for example in the treatment of cancer, or the need to delivery an active compound in a very selective area of the body, at the organ or cellular level, more specific triggers may be used. Among them, exogenous triggers are of particular interest as they can be activated directly by clinicians, on demand. Many different external stimuli have been developed, the most popular being temperature, magnetic/electric fields, hyperthermia, or ultrasound. In addition, light is presenting increasing attention and presents many advantages as a trigger for drug delivery systems. Its application is instantaneous, easy to control spatially and temporally, cheap to produce and noninvasive. This review focused on light-sensitive drug delivery systems at the molecular and macromolecular level, with the aim of understanding the underlying mechanisms involved in the release. Our goal was also to provide a wide overview of the recent advances performed in the field in order to be able to choose the best molecules and systems for future experiments. The different molecular and physical mechanisms induced by light irradiation have been reviewed, that is, which molecules and systems are involved in light-sensitive DDS and what is the effect of light on them. Thus, the different physical mechanisms are light energy converted into heat thanks to nanoheaters, upconversion, and the different molecular mechanisms involved are cleavage, isomerization and hydrophobicity / hydrophilicity switch. The property changes at the molecular level induced macromolecular effects 
on DDS structure such as destabilization, shrinkage, permeabilization and pore opening. This review demonstrated the potential of light-sensitive DDS, and promising results in vitro and in vivo for drug delivery are presented. The most promising candidates from our point of view are NIR sensitive systems such as those combining upconverting nanoparticles with UV sensitive molecules such as ONB or photocleavable coumarin groups. Several drug delivery applications have been successfully developed and therapeutic drug or protein release in vitro has been shown. Equally, nanoheaters such as gold nanoparticles combined with temperature responsive polymers such as pNIPAAM are an interesting and advanced approach, which has already been used in vivo. Before use for clinical trials, light sensitive DDS have to face several challenges that are common to all DDS, such as the demonstration of the non-toxic character of novel compounds used, the improved targeting and reduced unwanted drug leakage. More specifically for light sensitive DDS, light depth penetration should be large enough to reach some organs.

However, it is still difficult to reach internal organs even with IR. This field is still emerging and some challenges still need to be addressed, the most important being the design of efficient systems that can be activated in the NIR region, allowing for a deeper tissue penetration. This implies finding functional molecules with enhanced light absorption and quantum yield/efficiency of photoconversion and/or seeking different and less energy-demanding mechanisms to effect switching and consequently perturb the drug carrier.

\section{Acknowledgement}

This work is financially supported by a research grant from the Agence National de Recherche (N ANR-14-CE16-0015).

\section{Conflict of interest}

The authors declare no financial interest.

\section{$\underline{\text { References }}$}

[1] F. Alexis, E. Pridgen, L.K. Molnar, O.C. Farokhzad, Factors Affecting the Clearance and Biodistribution of Polymeric Nanoparticles, Mol. Pharm. 5 (2008) 505515. doi:10.1021/mp800051m.

[2] D.-Z. Liu, W.-Y. Chen, L.-M. Tasi, S.-P. Yang, Microcalorimetric and shear studies on the effects of cholesterol on the physical stability of lipid vesicles, Colloids Surf. Physicochem. Eng. Asp. 172 (2000) 57-67. doi:10.1016/S09277757(00)00560-4.

[3] C. Tw, H. Gh, C. Wk, L. Cj, The effects of plasma and shear force on the stability of liposome encapsulated hemoglobin - in vitro testing, J. Chin. Inst. Chem. Eng. 28 (1997) 407-413.

[4] S. Mura, J. Nicolas, P. Couvreur, Stimuli-responsive nanocarriers for drug delivery, Nat. Mater. 12 (2013) 991-1003. doi:10.1038/nmat3776.

[5] P.J. Kolari, Penetration of unfocused laser light into the skin, Arch. Dermatol. Res. 277 (1985) 342-344. 
[6] F.H. Mustafa, M.S. Jaafar, Comparison of wavelength-dependent penetration depths of lasers in different types of skin in photodynamic therapy, Indian J. Phys. 87 (2013) 203-209.

[7] D. Barolet, F. Christiaens, M.R. Hamblin, Infrared and Skin: Friend or Foe, J. Photochem. Photobiol. B. 155 (2016) 78-85. doi:10.1016/j.jphotobiol.2015.12.014.

[8] H. Piazena, W. Pittermann, W. Müller, K. Jung, D.K. Kelleher, T. Herrling, P. Meffert, R. Uebelhack, M. Kietzmann, Effects of water-filtered infrared-A and of heat on cell death, inflammation, antioxidative potential and of free radical formation in viable skin - First results, J. Photochem. Photobiol. B. 138 (2014) 347-354. doi:10.1016/j.jphotobiol.2014.06.007.

[9] P.M. Clement, G. Daniel, M. Trelles, Optimising the design of a broad-band light source for the treatment of skin, J. Cosmet. Laser Ther. 7 (2005) 177-189. doi:10.1080/14764170500344575.

[10] M.R. Hussein, Ultraviolet radiation and skin cancer: molecular mechanisms, J. Cutan. Pathol. 32 (2005) 191-205. doi:10.1111/j.0303-6987.2005.00281.x.

[11] M. Meinhardt, R. Krebs, A. Anders, U. Heinrich, H. Tronnier, Wavelengthdependent penetration depths of ultraviolet radiation in human skin, J. Biomed. Opt. 13 (2008) 044030. doi:10.1117/1.2957970.

[12] S.R. Sershen, S.L. Westcott, N.J. Halas, J.L. West, Temperature-sensitive polymer-nanoshell composites for photothermally modulated drug delivery, J.

Biomed. Mater. Res. 51 (2000) 293-298. doi:10.1002/10974636(20000905)51:3<293::AID-JBM1>3.0.CO;2-T.

[13] S. Huang, J. Liu, Q. He, H. Chen, J. Cui, S. Xu, Y. Zhao, C. Chen, L. Wang, Smart $\mathrm{Cu} 1.75 \mathrm{~S}$ nanocapsules with high and stable photothermal efficiency for NIR photo-triggered drug release, Nano Res. 8 (2015) 4038-4047. doi:10.1007/s12274015-0905-9.

[14] M.S. Yavuz, Y. Cheng, J. Chen, C.M. Cobley, Q. Zhang, M. Rycenga, J. Xie, C. Kim, A.G. Schwartz, L.V. Wang, Y. Xia, Gold nanocages covered by smart polymers for controlled release with near-infrared light, Nat. Mater. 8 (2009) 935939. doi:10.1038/nmat2564.

[15] J. Yang, J. Lee, J. Kang, S.J. Oh, H.-J. Ko, J.-H. Son, K. Lee, J.-S. Suh, Y.-M. Huh, S. Haam, Smart Drug-Loaded Polymer Gold Nanoshells for Systemic and Localized Therapy of Human Epithelial Cancer, Adv. Mater. 21 (2009) 4339-4342. doi:10.1002/adma.200900334.

[16] Basuki Johan S., Qie Fengxiang, Mulet Xavier, Suryadinata Randy, Vashi Aditya V., Peng Yong Y., Li Lingli, Hao Xiaojuan, Tan Tianwei, Hughes Timothy C., Photo-Modulated Therapeutic Protein Release from a Hydrogel Depot Using Visible Light, Angew. Chem. 129 (2016) 986-991. doi:10.1002/ange.201610618.

[17] G. Chen, H. Qiu, P.N. Prasad, X. Chen, Upconversion Nanoparticles: Design, Nanochemistry, and Applications in Theranostics, Chem. Rev. 114 (2014) 51615214. doi:10.1021/cr400425h.

[18] B. Yan, J.-C. Boyer, N.R. Branda, Y. Zhao, Near-Infrared Light-Triggered Dissociation of Block Copolymer Micelles Using Upconverting Nanoparticles, J. Am. Chem. Soc. 133 (2011) 19714-19717. doi:10.1021/ja209793b.

[19] S. Chen, Y. Gao, Z. Cao, B. Wu, L. Wang, H. Wang, Z. Dang, G. Wang, Nanocomposites of Spiropyran-Functionalized Polymers and Upconversion Nanoparticles for Controlled Release Stimulated by Near-Infrared Light and $\mathrm{pH}$, Macromolecules. 49 (2016) 7490-7496. doi:10.1021/acs.macromol.6b01760. [20] G. Jalani, R. Naccache, D.H. Rosenzweig, L. Haglund, F. Vetrone, M. Cerruti, Photocleavable hydrogel coated upconverting nanoparticles: a multifunctional 
theranostic platform for NIR imaging and on-demand macromolecular delivery, J. Am. Chem. Soc. (2015). doi:10.1021/jacs.5b12357.

[21] X. Huang, P.K. Jain, I.H. El-Sayed, M.A. El-Sayed, Plasmonic photothermal therapy (PPTT) using gold nanoparticles, Lasers Med. Sci. 23 (2007) 217. doi:10.1007/s10103-007-0470-x.

[22] H.H. Richardson, M.T. Carlson, P.J. Tandler, P. Hernandez, A.O. Govorov, Experimental and Theoretical Studies of Light-to-Heat Conversion and Collective Heating Effects in Metal Nanoparticle Solutions, Nano Lett. 9 (2009) 1139-1146. doi:10.1021/nl8036905.

[23] S. Linic, U. Aslam, C. Boerigter, M. Morabito, Photochemical transformations on plasmonic metal nanoparticles, Nat. Mater. 14 (2015) 567-576.

doi:10.1038/nmat4281.

[24] C. Voisin, N. Del Fatti, D. Christofilos, F. Vallée, Ultrafast Electron Dynamics and Optical Nonlinearities in Metal Nanoparticles, J. Phys. Chem. B. 105 (2001) 2264-2280. doi:10.1021/jp0038153.

[25] Y. Xia, Y. Xiong, B. Lim, S.E. Skrabalak, Shape-controlled synthesis of metal nanocrystals: simple chemistry meets complex physics?, Angew. Chem. Int. Ed Engl. 48 (2009) 60-103. doi:10.1002/anie.200802248.

[26] J. Conde, G. Doria, P. Baptista, Noble Metal Nanoparticles Applications in Cancer, J. Drug Deliv. (2012). doi:10.1155/2012/751075.

[27] G.P. Robbins, M. Jimbo, J. Swift, M.J. Therien, D.A. Hammer, I.J. Dmochowski, Photoinitiated Destruction of Composite Porphyrin-Protein Polymersomes, J. Am. Chem. Soc. 131 (2009) 3872-3874. doi:10.1021/ja808586q. [28] A. Suzuki, T. Tanaka, Phase transition in polymer gels induced by visible light, Nature. 346 (1990) 345-347. doi:10.1038/346345a0.

[29] W. Yin, L. Yan, J. Yu, G. Tian, L. Zhou, X. Zheng, X. Zhang, Y. Yong, J. Li, Z. Gu, Y. Zhao, High-Throughput Synthesis of Single-Layer MoS2 Nanosheets as a Near-Infrared Photothermal-Triggered Drug Delivery for Effective Cancer Therapy, ACS Nano. 8 (2014) 6922-6933. doi:10.1021/nn501647j.

[30] B. Tian, C. Wang, S. Zhang, L. Feng, Z. Liu, Photothermally Enhanced Photodynamic Therapy Delivered by Nano-Graphene Oxide, ACS Nano. 5 (2011) 7000-7009. doi:10.1021/nn201560b.

[31] K. Yang, L. Feng, Z. Liu, Stimuli responsive drug delivery systems based on nano-graphene for cancer therapy, Adv. Drug Deliv. Rev. 105 (2016) 228-241. doi:10.1016/j.addr.2016.05.015.

[32] L. Gong, L. Yan, R. Zhou, J. Xie, W. Wu, Z. Gu, Two-dimensional transition metal dichalcogenide nanomaterials for combination cancer therapy, J. Mater. Chem. B. 5 (2017) 1873-1895. doi:10.1039/C7TB00195A.

[33] Z. Su, S. Zhu, A.D. Donkor, C. Tzoganakis, J.F. Honek, Controllable Delivery of Small-Molecule Compounds to Targeted Cells Utilizing Carbon Nanotubes, J. Am. Chem. Soc. 133 (2011) 6874-6877. doi:10.1021/ja1084282.

[34] M. Martincic, G. Tobias, Filled carbon nanotubes in biomedical imaging and drug delivery, Expert Opin. Drug Deliv. 12 (2015) 563-581.

doi:10.1517/17425247.2015.971751.

[35] X. Huang, W. Zhang, G. Guan, G. Song, R. Zou, J. Hu, Design and Functionalization of the NIR-Responsive Photothermal Semiconductor Nanomaterials for Cancer Theranostics, Acc. Chem. Res. 50 (2017) 2529-2538.

doi:10.1021/acs.accounts.7b00294.

[36] N. Kamaly, Z. Xiao, P.M. Valencia, A.F. Radovic-Moreno, O.C. Farokhzad, Targeted polymeric therapeutic nanoparticles: design, development and clinical 
translation, Chem. Soc. Rev. 41 (2012) 2971-3010. doi:10.1039/c2cs15344k. [37] Y. Wu, K. Wang, S. Huang, C. Yang, M. Wang, Near-Infrared LightResponsive Semiconductor Polymer Composite Hydrogels: Spatial/TemporalControlled Release via a Photothermal "Sponge" Effect, ACS Appl. Mater. Interfaces. 9 (2017) 13602-13610. doi:10.1021/acsami.7b01016.

[38] A.M. Smith, M.C. Mancini, S. Nie, Bioimaging: Second window for in vivo imaging, Nat. Nanotechnol. 4 (2009) 710-711. doi:10.1038/nnano.2009.326.

[39] Z. Cao, L. Feng, G. Zhang, J. Wang, S. Shen, D. Li, X. Yang, Semiconducting polymer-based nanoparticles with strong absorbance in NIR-II window for in vivo photothermal therapy and photoacoustic imaging, Biomaterials. 155 (2018) 103-111. doi:10.1016/j.biomaterials.2017.11.016.

[40] M.A. Ward, T.K. Georgiou, Thermoresponsive polymers for biomedical applications, Polymers. 3 (2011) 1215-1242.

[41] M. Karimi, P. Sahandi Zangabad, A. Ghasemi, M. Amiri, M. Bahrami, H. Malekzad, H. Ghahramanzadeh Asl, Z. Mahdieh, M. Bozorgomid, A. Ghasemi, M.R. Rahmani Taji Boyuk, M.R. Hamblin, Temperature-Responsive Smart Nanocarriers for Delivery Of Therapeutic Agents: Applications and Recent Advances, ACS Appl. Mater. Interfaces. 8 (2016) 21107-21133. doi:10.1021/acsami.6b00371. [42] F. Wang, D. Banerjee, Y. Liu, X. Chen, X. Liu, Upconversion nanoparticles in biological labeling, imaging, and therapy, Analyst. 135 (2010) 1839-1854. doi:10.1039/COAN00144A.

[43] X. Li, F. Zhang, D. Zhao, Lab on upconversion nanoparticles: optical properties and applications engineering via designed nanostructure, Chem. Soc. Rev. 44 (2015) 1346-1378. doi:10.1039/C4CS00163J.

[44] J. Olejniczak, C.-J. Carling, A. Almutairi, Photocontrolled release using onephoton absorption of visible or NIR light, J. Controlled Release. 219 (2015) 18-30. doi:10.1016/j.jconrel.2015.09.030.

[45] P. Klán, T. Šolomek, C.G. Bochet, A. Blanc, R. Givens, M. Rubina, V. Popik, A. Kostikov, J. Wirz, Photoremovable Protecting Groups in Chemistry and Biology: Reaction Mechanisms and Efficacy, Chem. Rev. 113 (2013) 119-191. doi:10.1021/cr300177k.

[46] B. Schade, V. Hagen, R. Schmidt, R. Herbrich, E. Krause, T. Eckardt, J. Bendig, Deactivation Behavior and Excited-State Properties of (Coumarin-4-yl)methyl Derivatives. 1. Photocleavage of (7-Methoxycoumarin-4-yl)methyl-Caged Acids with Fluorescence Enhancement, J. Org. Chem. 64 (1999) 9109-9117. doi:10.1021/jo9910233.

[47] P. Pitter, Determination of biological degradability of organic substances, Water Res. 10 (1976) 231-235. doi:10.1016/0043-1354(76)90132-9.

[48] S. des experts-chimistes de France, Annales des falsifications, de l'expertise chimique et toxicologique, La Société, 1983.

[49] X. Hu, J. Tian, T. Liu, G. Zhang, S. Liu, Photo-Triggered Release of Caged Camptothecin Prodrugs from Dually Responsive Shell Cross-Linked Micelles, Macromolecules. 46 (2013) 6243-6256. doi:10.1021/ma400691j.

[50] Z. Zhang, H. Hatta, T. Ito, S. Nishimoto, Synthesis and photochemical properties of photoactivated antitumor prodrugs releasing 5-fluorouracil, Org. Biomol. Chem. 3 (2005) 592-596. doi:10.1039/B417734G.

[51] C. Wang, G. Zhang, G. Liu, J. Hu, S. Liu, Photo- and thermo-responsive multicompartment hydrogels for synergistic delivery of gemcitabine and doxorubicin, J. Controlled Release. 259 (2017) 149-159. doi:10.1016/j.jconrel.2016.11.007. [52] W.-J. Zhang, C.-Y. Hong, C.-Y. Pan, Efficient Fabrication of Photosensitive 
Polymeric Nano-objects via an Ingenious Formulation of RAFT Dispersion Polymerization and Their Application for Drug Delivery, Biomacromolecules. 18 (2017) 1210-1217. doi:10.1021/acs.biomac.6b01887.

[53] G. Liu, X. Wang, J. Hu, G. Zhang, S. Liu, Self-Immolative Polymersomes for High-Efficiency Triggered Release and Programmed Enzymatic Reactions, J. Am. Chem. Soc. 136 (2014) 7492-7497. doi:10.1021/ja5030832.

[54] M. Álvarez, J.M. Alonso, O. Filevich, M. Bhagawati, R. Etchenique, J. Piehler, A. del Campo, Modulating Surface Density of Proteins via Caged Surfaces and Controlled Light Exposure, Langmuir. 27 (2011) 2789-2795. doi:10.1021/la104511x. [55] A. Specht, M. Goeldner, 1-(o-Nitrophenyl)-2,2,2-trifluoroethyl Ether Derivatives as Stable and Efficient Photoremovable Alcohol-Protecting Groups, Angew. Chem. Int. Ed. 43 (2004) 2008-2012. doi:10.1002/anie.200353247.

[56] E. Reichmanis, B.C. Smith, R. Gooden, O-nitrobenzyl photochemistry: Solution vs. solid-state behavior, J. Polym. Sci. Polym. Chem. Ed. 23 (1985) 1-8. doi:10.1002/pol.1985.170230101.

[57] R.E. Kohman, S.S. Cha, H.-Y. Man, X. Han, Light-Triggered Release of Bioactive Molecules from DNA Nanostructures, Nano Lett. 16 (2016) 2781-2785. doi:10.1021/acs.nanolett.6b00530.

[58] M.M. Mahmoodi, D. Abate-Pella, T.J. Pundsack, C.C. Palsuledesai, P.C. Goff, D.A. Blank, M.D. Distefano, Nitrodibenzofuran: A One- and Two-Photon Sensitive Protecting Group That Is Superior to Brominated Hydroxycoumarin for Thiol Caging in Peptides, J. Am. Chem. Soc. 138 (2016) 5848-5859. doi:10.1021/jacs.5b11759. [59] K.A. Mosiewicz, L. Kolb, A.J. van der Vlies, M.M. Martino, P.S. Lienemann, J.A. Hubbell, M. Ehrbar, M.P. Lutolf, In situ cell manipulation through enzymatic hydrogel photopatterning, Nat. Mater. 12 (2013) 1072-1078. doi:10.1038/nmat3766. [60] N. Kretschy, A.-K. Holik, V. Somoza, K.-P. Stengele, M.M. Somoza, NextGeneration o-Nitrobenzyl Photolabile Groups for Light-Directed Chemistry and Microarray Synthesis, Angew. Chem. Int. Ed. 54 (2015) 8555-8559. doi:10.1002/anie.201502125.

[61] G.J. Finn, B. Creaven, D.A. Egan, Study of the in vitro cytotoxic potential of natural and synthetic coumarin derivatives using human normal and neoplastic skin cell lines, Melanoma Res. 11 (2001) 461.

[62] K.C. Fylaktakidou, D.J. Hadjipavlou-Litina, K.E. Litinas, D.N. Nicolaides, Natural and Synthetic Coumarin Derivatives with Anti-Inflammatory / Antioxidant Activities, (2004). doi:info:doi/10.2174/1381612043382710.

[63] W.A. Velema, J.P. van der Berg, W. Szymanski, A.J.M. Driessen, B.L. Feringa, Orthogonal Control of Antibacterial Activity with Light, ACS Chem. Biol. 9 (2014) 1969-1974. doi:10.1021/cb500313f.

[64] Q. Huang, C. Bao, W. Ji, Q. Wang, L. Zhu, Photocleavable coumarin crosslinkers based polystyrene microgels: phototriggered swelling and release, J. Mater. Chem. 22 (2012) 18275-18282. doi:10.1039/C2JM33789D.

[65] W. Ji, N. Li, D. Chen, X. Qi, W. Sha, Y. Jiao, Q. Xu, J. Lu, Coumarincontaining photo-responsive nanocomposites for NIR light-triggered controlled drug release via a two-photon process, J. Mater. Chem. B. 1 (2013) 5942-5949. doi:10.1039/C3TB21206H.

[66] C. Hang, Y. Zou, Y. Zhong, Z. Zhong, F. Meng, NIR and UV-responsive degradable hyaluronic acid nanogels for CD44-targeted and remotely triggered intracellular doxorubicin delivery, Colloids Surf. B Biointerfaces. 158 (2017) 547-555. doi:10.1016/j.colsurfb.2017.07.041.

[67] L. Fournier, I. Aujard, T. Le Saux, S. Maurin, S. Beaupierre, J.-B. Baudin, L. 
Jullien, Coumarinylmethyl Caging Groups with Redshifted Absorption, Chem. - Eur. J. 19 (2013) 17494-17507. doi:10.1002/chem.201302630.

[68] S. Karthik, A. Jana, M. Selvakumar, Y. Venkatesh, A. Paul, S.S. Shah, N.D.P. Singh, Coumarin polycaprolactone polymeric nanoparticles: light and tumor microenvironment activated cocktail drug delivery, J. Mater. Chem. B. 5 (2017) 1734-1741. doi:10.1039/C6TB02944B.

[69] L. Zayat, M.G. Noval, J. Campi, C.I. Calero, D.J. Calvo, R. Etchenique, A New Inorganic Photolabile Protecting Group for Highly Efficient Visible Light GABA Uncaging, ChemBioChem. 8 (2007) 2035-2038. doi:10.1002/cbic.200700354.

[70] N.Z. Knezevic, B.G. Trewyn, V.S.-Y. Lin, Functionalized mesoporous silica nanoparticle-based visible light responsive controlled release delivery system, Chem. Commun. 47 (2011) 2817-2819. doi:10.1039/C0CC04424E.

[71] W. Sun, M. Parowatkin, W. Steffen, H.-J. Butt, V. Mailänder, S. Wu, Ruthenium-Containing Block Copolymer Assemblies: Red-Light-Responsive Metallopolymers with Tunable Nanostructures for Enhanced Cellular Uptake and Anticancer Phototherapy, Adv. Healthc. Mater. 5 (2016) 467-473. doi:10.1002/adhm.201500827.

[72] T. Slanina, P. Shrestha, E. Palao, D. Kand, J.A. Peterson, A.S. Dutton, N. Rubinstein, R. Weinstain, A.H. Winter, P. Klán, In Search of the Perfect Photocage: Structure-Reactivity Relationships in meso-Methyl BODIPY Photoremovable Protecting Groups, J. Am. Chem. Soc. 139 (2017) 15168-15175. doi:10.1021/jacs.7b08532.

[73] P.P. Goswami, A. Syed, C.L. Beck, T.R. Albright, K.M. Mahoney, R. Unash, E.A. Smith, A.H. Winter, BODIPY-Derived Photoremovable Protecting Groups Unmasked with Green Light, J. Am. Chem. Soc. 137 (2015) 3783-3786. doi:10.1021/jacs.5b01297.

[74] J. Jiang, X. Tong, Y. Zhao, A New Design for Light-Breakable Polymer Micelles, J. Am. Chem. Soc. 127 (2005) 8290-8291. doi:10.1021/ja0521019. [75] T. Furuta, H. Torigai, T. Osawa, M. Iwamura, New photochemically labile protecting group for phosphates, Chem. Lett. 22 (1993) 1179-1182.

[76] H. Du, R.-C.A. Fuh, J. Li, L.A. Corkan, J.S. Lindsey, PhotochemCAD: A Computer-Aided Design and Research Tool in Photochemistry, Photochem. Photobiol. 68 (1998) 141-142. doi:10.1111/j.1751-1097.1998.tb02480.x.

[77] A. Jana, M. Ikbal, N.D.P. Singh, Perylen-3-ylmethyl: fluorescent photoremovable protecting group (FPRPG) for carboxylic acids and alcohols, Tetrahedron. 68 (2012) 1128-1136. doi:10.1016/j.tet.2011.11.074.

[78] M.A. Ayer, S. Schrettl, S. Balog, Y.C. Simon, C. Weder, Light-responsive azocontaining organogels, Soft Matter. 13 (2017) 4017-4023.

doi:10.1039/C7SM00601B.

[79] C.-J. Carling, J. Olejniczak, A. Foucault-Collet, G. Collet, M.L. Viger, V.A.N. Huu, B.M. Duggan, A. Almutairi, Efficient red light photo-uncaging of active molecules in water upon assembly into nanoparticles, Chem. Sci. 7 (2016) 23922398. doi:10.1039/C5SC03717D.

[80] J. Broichhagen, J.A. Frank, D. Trauner, A Roadmap to Success in Photopharmacology, Acc. Chem. Res. 48 (2015) 1947-1960.

doi:10.1021/acs.accounts.5b00129.

[81] G.S. Hartley, The Cis-form of Azobenzene, Nature. 140 (1937) 281. doi:10.1038/140281a0.

[82] D. Bléger, J. Schwarz, A.M. Brouwer, S. Hecht, o-Fluoroazobenzenes as Readily Synthesized Photoswitches Offering Nearly Quantitative Two-Way 
Isomerization with Visible Light, J. Am. Chem. Soc. 134 (2012) 20597-20600. doi:10.1021/ja310323y.

[83] S. Subhas, B. Amirhossein, D. Ming-xin, W.G. Andrew, Photoswitching of ortho-Substituted Azonium lons by Red Light in Whole Blood, Angew. Chem. Int. Ed. 52 (2013) 14127-14130.

[84] S. Samanta, A.A. Beharry, O. Sadovski, T.M. McCormick, A. Babalhavaeji, V. Tropepe, G.A. Woolley, Photoswitching Azo Compounds in Vivo with Red Light, J. Am. Chem. Soc. 135 (2013) 9777-9784. doi:10.1021/ja402220t.

[85] M. Dong, A. Babalhavaeji, S. Samanta, A.A. Beharry, G.A. Woolley, RedShifting Azobenzene Photoswitches for in Vivo Use, Acc. Chem. Res. 48 (2015) 2662-2670. doi:10.1021/acs.accounts.5b00270.

[86] E. Mabrouk, D. Cuvelier, F. Brochard-Wyart, P. Nassoy, M.-H. Li, Bursting of sensitive polymersomes induced by curling, Proc. Natl. Acad. Sci. 106 (2009) 72947298. doi:10.1073/pnas.0813157106.

[87] O. Sadovski, A.A. Beharry, F. Zhang, G.A. Woolley, Spectral Tuning of Azobenzene Photoswitches for Biological Applications, Angew. Chem. Int. Ed. 48 (2009) 1484-1486. doi:10.1002/anie.200805013.

[88] A. Rullo, A. Reiner, A. Reiter, D. Trauner, E.Y. Isacoff, G.A. Woolley, Long wavelength optical control of glutamate receptor ion channels using a tetra-orthosubstituted azobenzene derivative, Chem. Commun. 50 (2014) 14613-14615. doi:10.1039/C4CC06612J.

[89] D. Wang, S. Wu, Red-Light-Responsive Supramolecular Valves for Photocontrolled Drug Release from Mesoporous Nanoparticles, Langmuir. 32 (2016) 632-636. doi:10.1021/acs.langmuir.5b04399.

[90] R. Tong, H.D. Hemmati, R. Langer, D.S. Kohane, Photoswitchable Nanoparticles for Triggered Tissue Penetration and Drug Delivery, J. Am. Chem. Soc. 134 (2012) 8848-8855. doi:10.1021/ja211888a.

[91] A.P. Goodwin, J.L. Mynar, Y. Ma, G.R. Fleming, J.M.J. Fréchet, Synthetic Micelle Sensitive to IR Light via a Two-Photon Process, J. Am. Chem. Soc. 127 (2005) 9952-9953. doi:10.1021/ja0523035.

[92] Q. Li, Z. Cao, G. Wang, Diazonaphthoquinone-based amphiphilic polymer assemblies for NIR/UV light- and $\mathrm{pH}$-responsive controlled release, Polym. Chem. 9 (2018) 463-471. doi:10.1039/C7PY01822C.

[93] S. Helmy, F.A. Leibfarth, S. Oh, J.E. Poelma, C.J. Hawker, J. Read de Alaniz, Photoswitching Using Visible Light: A New Class of Organic Photochromic

Molecules, J. Am. Chem. Soc. 136 (2014) 8169-8172. doi:10.1021/ja503016b.

[94] S.O. Poelma, S.S. Oh, S. Helmy, A.S. Knight, G.L. Burnett, H.T. Soh, C.J. Hawker, J.R. de Alaniz, Controlled drug release to cancer cells from modular onephoton visible light-responsive micellar system, Chem. Commun. 52 (2016) 1052510528. doi:10.1039/C6CC04127B.

[95] G.-Y. Liu, C.-J. Chen, D.-D. Li, S.-S. Wang, J. Ji, Near-infrared light-sensitive micelles for enhanced intracellular drug delivery, J. Mater. Chem. 22 (2012) 1686516871. doi:10.1039/C2JM00045H.

[96] S. Son, E. Shin, B.-S. Kim, Light-Responsive Micelles of Spiropyran Initiated Hyperbranched Polyglycerol for Smart Drug Delivery, Biomacromolecules. 15 (2014) 628-634. doi:10.1021/bm401670t.

[97] C. Chen, G. Liu, X. Liu, S. Pang, C. Zhu, L. Lv, J. Ji, Photo-responsive, biocompatible polymeric micelles self-assembled from hyperbranched polyphosphate-based polymers, Polym. Chem. 2 (2011) 1389-1397. doi:10.1039/C1PY00094B. 
[98] R. Klajn, Spiropyran-based dynamic materials, Chem. Soc. Rev. 43 (2013) 148-184. doi:10.1039/C3CS60181A.

[99] L. Sun, B. Zhu, Y. Su, C.-M. Dong, Light-responsive linear-dendritic amphiphiles and their nanomedicines for NIR-triggered drug release, Polym. Chem. 5 (2014) 1605-1613. doi:10.1039/C3PY00533J.

[100] W. Kirmse, 100 Years of the Wolff Rearrangement, Eur. J. Org. Chem. 2002 (2002) 2193-2256. doi:10.1002/1099-0690(200207)2002:14<2193::AID-

EJOC2193>3.0.CO;2-D.

[101] J.I.K. Almstead, B. Urwyler, J. Wirz, Flash Photolysis of .alpha.-

Diazonaphthoquinones in Aqueous Solution: Determination of Rates and Equilibria for Keto-Enol Tautomerization of 1-Indene-3-carboxylic Acid, J. Am. Chem. Soc. 116 (1994) 954-960. doi:10.1021/ja00082a016.

[102] O. Rifaie-Graham, S. Ulrich, N.F.B. Galensowske, S. Balog, M. Chami, D. Rentsch, J.R. Hemmer, J.R. de Alaniz, L.F. Boesel, N. Bruns, Wavelength-Selective Light-Responsive DASA-Functionalized Polymersome Nanoreactors, J. Am. Chem. Soc. (2018). doi:10.1021/jacs.8b04511.

[103] J. Jiang, B. Qi, M. Lepage, Y. Zhao, Polymer Micelles Stabilization on Demand through Reversible Photo-Cross-Linking, Macromolecules. 40 (2007) 790-792. doi:10.1021/ma062493j.

[104] S.R. Trenor, A.R. Shultz, B.J. Love, T.E. Long, Coumarins in Polymers: From Light Harvesting to Photo-Cross-Linkable Tissue Scaffolds, Chem. Rev. 104 (2004) 3059-3078. doi:10.1021/cr030037c.

[105] M.V.S.N. Maddipatla, D. Wehrung, C. Tang, W. Fan, M.O. Oyewumi, T. Miyoshi, A. Joy, Photoresponsive Coumarin Polyesters That Exhibit Cross-Linking and Chain Scission Properties, Macromolecules. 46 (2013) 5133-5140. doi:10.1021/ma400584y.

[106] Y. Zheng, F.M. Andreopoulos, M. Micic, Q. Huo, S.M. Pham, R.M. Leblanc, A Novel Photoscissile Poly(ethylene glycol)-Based Hydrogel, Adv. Funct. Mater. 11 (2001) 37-40. doi:10.1002/1616-3028(200102)11:1<37::AID-ADFM37>3.0.CO;2-V. [107] Y. Sako, Y. Takaguchi, A photo-responsive hydrogelator having gluconamides at its peripheral branches, Org. Biomol. Chem. 6 (2008) 3843-3847.

doi:10.1039/B810900A.

[108] C. Zhu, C.J. Bettinger, Light-induced remodeling of physically crosslinked hydrogels using near-IR wavelengths, J. Mater. Chem. B. 2 (2014) 1613-1618. doi:10.1039/C3TB21689F.

[109] T. Nishimura, M. Takara, S. Mukai, S. Sawada, Y. Sasaki, K. Akiyoshi, A light sensitive self-assembled nanogel as a tecton for protein patterning materials, Chem. Commun. 52 (2016) 1222-1225. doi:10.1039/C5CC08416D.

[110] M.A. Azagarsamy, D.D. McKinnon, D.L. Alge, K.S. Anseth, Coumarin-Based Photodegradable Hydrogel: Design, Synthesis, Gelation, and Degradation Kinetics, ACS Macro Lett. 3 (2014) 515-519. doi:10.1021/mz500230p.

[111] D. Klinger, K. Landfester, Dual Stimuli-Responsive Poly(2-hydroxyethyl methacrylate-co-methacrylic acid) Microgels Based on Photo-Cleavable CrossLinkers: pH-Dependent Swelling and Light-Induced Degradation, Macromolecules. 44 (2011) 9758-9772. doi:10.1021/ma201706r.

[112] B.M. Discher, Y.Y. Won, D.S. Ege, J.C. Lee, F.S. Bates, D.E. Discher, D.A. Hammer, Polymersomes: tough vesicles made from diblock copolymers, Science. 284 (1999) 1143-1146.

[113] A. Blanazs, J. Madsen, G. Battaglia, A.J. Ryan, S.P. Armes, Mechanistic Insights for Block Copolymer Morphologies: How Do Worms Form Vesicles?, J. Am. 
Chem. Soc. 133 (2011) 16581-16587. doi:10.1021/ja206301a.

[114] E. Cabane, V. Malinova, W. Meier, Synthesis of Photocleavable Amphiphilic Block Copolymers: Toward the Design of Photosensitive Nanocarriers, Macromol. Chem. Phys. 211 (2010) 1847-1856. doi:10.1002/macp.201000151.

[115] S. Chen, F. Jiang, Z. Cao, G. Wang, Z.-M. Dang, Photo, pH, and thermo tripleresponsive spiropyran-based copolymer nanoparticles for controlled release, Chem. Commun. 51 (2015) 12633-12636. doi:10.1039/C5CC04087F.

[116] Y. Xue, J. Tian, W. Tian, P. Gong, J. Dai, X. Wang, Significant Fluorescence Enhancement of Spiropyran in Colloidal Dispersion and Its Light-Induced Size Tunability for Release Control, J. Phys. Chem. C. 119 (2015) 20762-20772. doi:10.1021/acs.jpcc.5b06905.

[117] Y. Zhao, Photocontrollable block copolymer micelles: what can we control?, J. Mater. Chem. 19 (2009) 4887-4895. doi:10.1039/B819968J.

[118] G. Wang, X. Tong, Y. Zhao, Preparation of Azobenzene-Containing Amphiphilic Diblock Copolymers for Light-Responsive Micellar Aggregates, Macromolecules. 37 (2004) 8911-8917. doi:10.1021/ma048416a.

[119] S. Pautot, B.J. Frisken, D.A. Weitz, Engineering asymmetric vesicles, Proc. Natl. Acad. Sci. 100 (2003) 10718-10721. doi:10.1073/pnas.1931005100.

[120] Peyret Ariane, Ibarboure Emmanuel, Tron Arnaud, Beauté Louis, Rust Ruben, Sandre Olivier, McClenaghan Nathan D., Lecommandoux Sebastien, Polymersome Popping by Light-Induced Osmotic Shock under Temporal, Spatial, and Spectral Control, Angew. Chem. Int. Ed. 56 (2017) 1566-1570. doi:10.1002/anie.201609231. [121] R.T. Chacko, J. Ventura, J. Zhuang, S. Thayumanavan, Polymer nanogels: A versatile nanoscopic drug delivery platform, Adv. Drug Deliv. Rev. 64 (2012) 836851. doi:10.1016/j.addr.2012.02.002.

[122] Q. Huang, C. Bao, W. Ji, Q. Wang, L. Zhu, Photocleavable coumarin crosslinkers based polystyrene microgels: phototriggered swelling and release, J. Mater. Chem. 22 (2012) 18275-18282. doi:10.1039/C2JM33789D.

[123] B.A. Badeau, M.P. Comerford, C.K. Arakawa, J.A. Shadish, C.A. DeForest, Engineered modular biomaterial logic gates for environmentally triggered therapeutic delivery, Nat. Chem. 10 (2018) 251-258. doi:10.1038/nchem.2917.

[124] S. Hocine, M.-H. Li, Thermoresponsive self-assembled polymer colloids in water, Soft Matter. 9 (2013) 5839-5861. doi:10.1039/C3SM50428J.

[125] D. Roy, W.L.A. Brooks, B.S. Sumerlin, New directions in thermoresponsive polymers, Chem. Soc. Rev. 42 (2013) 7214-7243. doi:10.1039/C3CS35499G.

[126] W. Agut, A. Brûlet, D. Taton, S. Lecommandoux, Thermoresponsive Micelles from Jeffamine-b-poly(l-glutamic acid) Double Hydrophilic Block Copolymers, Langmuir. 23 (2007) 11526-11533. doi:10.1021/la701482w.

[127] Garanger Elisabeth, Lecommandoux Sébastien, Towards Bioactive Nanovehicles Based on Protein Polymers, Angew. Chem. Int. Ed. 51 (2012) 30603062. doi:10.1002/anie.201107734.

[128] M. Beija, J.-D. Marty, M. Destarac, Thermoresponsive poly(N-vinyl caprolactam)-coated gold nanoparticles: sharp reversible response and easy tunability, Chem. Commun. 47 (2011) 2826-2828. doi:10.1039/C0CC05184E. [129] H.K. Makadia, S.J. Siegel, Poly Lactic-co-Glycolic Acid (PLGA) as Biodegradable Controlled Drug Delivery Carrier, Polymers. 3 (2011) 1377-1397. doi:10.3390/polym3031377.

[130] J.M. Anderson, M.S. Shive, Biodegradation and biocompatibility of PLA and PLGA microspheres, Adv. Drug Deliv. Rev. 28 (1997) 5-24. doi:10.1016/S0169409X(97)00048-3. 
[131] S.-M. Lee, H. Park, J.-W. Choi, Y.N. Park, C.-O. Yun, K.-H. Yoo, Multifunctional Nanoparticles for Targeted Chemophotothermal Treatment of Cancer Cells, Angew. Chem. Int. Ed. 50 (2011) 7581-7586. doi:10.1002/anie.201101783.

[132] C. Sanson, O. Diou, J. Thevenot, E. Ibarboure, A. Soum, A. Brûlet, S. Miraux, E. Thiaudière, S. Tan, A. Brisson, V. Dupuis, O. Sandre, S. Lecommandoux, Doxorubicin Loaded Magnetic Polymersomes: Theranostic Nanocarriers for MR Imaging and Magneto-Chemotherapy, LLB Highlights 2011. (2012) 48-49. [133] T. Trantidou, M. Friddin, Y. Elani, N.J. Brooks, R.V. Law, J.M. Seddon, O. Ces, Engineering Compartmentalized Biomimetic Micro- and Nanocontainers, ACS Nano. 11 (2017) 6549-6565. doi:10.1021/acsnano.7b03245.

[134] P. Shrager, S.Y. Chiu, J.M. Ritchie, Voltage-dependent sodium and potassium channels in mammalian cultured Schwann cells, Proc. Natl. Acad. Sci. 82 (1985) 948-952.

[135] S. Geng, Y. Wang, L. Wang, T. Kouyama, T. Gotoh, S. Wada, J.-Y. Wang, A Light-Responsive Self-Assembly Formed by a Cationic Azobenzene Derivative and SDS as a Drug Delivery System, Sci. Rep. 7 (2017) 39202. doi:10.1038/srep39202. [136] X. Wang, J. Hu, G. Liu, J. Tian, H. Wang, M. Gong, S. Liu, Reversibly Switching Bilayer Permeability and Release Modules of Photochromic Polymersomes Stabilized by Cooperative Noncovalent Interactions, J. Am. Chem. Soc. 137 (2015) 15262-15275. doi:10.1021/jacs.5b10127.

[137] K. Niikura, N. Iyo, Y. Matsuo, H. Mitomo, K. ljiro, Sub-100 nm gold nanoparticle vesicles as a drug delivery carrier enabling rapid drug release upon light irradiation, ACS Appl. Mater. Interfaces. 5 (2013) 3900-3907.

doi:10.1021/am400590m. 